\title{
Optimal noise in a stochastic model for local search
}

\author{
J. Noetel, ${ }^{1}$ V. L. S. Freitas, ${ }^{2}$ E. E. N. Macau, ${ }^{2,3}$ and L. Schimansky-Geier ${ }^{1,4}$ \\ ${ }^{1}$ Institute of Physics, Humboldt University at Berlin, Newtonstraße 15, 12489 Berlin, Germany \\ ${ }^{2}$ National Institute for Space Research, 12227-010 São José dos Campos, São Paulo, Brazil \\ ${ }^{3}$ Federal University of Sao Paulo, 12247-014 São José dos Campos, São Paulo, Brazil \\ ${ }^{4}$ Berlin Bernstein Center for Computational Neuroscience, Humboldt University at Berlin, Unter den Linden 6, 10099 Berlin, Germany
}

(Received 7 February 2018; revised manuscript received 31 May 2018; published 27 August 2018)

\begin{abstract}
We develop a prototypical stochastic model for a local search around a given home. The stochastic dynamic model is motivated by experimental findings of the motion of a fruit fly around a given spot of food but will generally describe the local search behavior. The local search consists of a sequence of two epochs. In the first the searcher explores new space around the home, whereas it returns to the home during the second epoch. In the proposed two-dimensional model both tasks are described by the same stochastic dynamics. The searcher moves with constant speed and its angular dynamics is driven by a symmetric $\alpha$-stable noise source. The latter stands for the uncertainty to decide the new direction of motion. The main ingredient of the model is the nonlinear interaction dynamics of the searcher with its home. In order to determine the new heading direction, the searcher has to know the actual angles of its position to the home and of the heading vector. A bound state to the home is realized by a permanent switch of a repulsive and attractive forcing of the heading direction from the position direction corresponding to search and return epochs. Our investigation elucidates the analytic tractability of the deterministic and stochastic dynamics. Noise transforms the conservative deterministic dynamics into a dissipative one of the moments. The noise enables a faster finding of a target distinct from the home with optimal intensity. This optimal situation is related to the noise-dependent relaxation time. It is uniquely defined for all $\alpha$ and distinguishes between the stochastic dynamics before and after its value. For times large compared to this, we derive the corresponding Smoluchowski equation and find diffusive spreading of the searcher in the space. We report on the qualitative agreement with the experimentally observed spatial distribution, noisy oscillatory return times, and spatial autocorrelation function of the fruit fly. However, as a result of its simplicity, the model aims to reproduce the local search behavior of other units during their exploration of surrounding space and their quasiperiodic return to a home.
\end{abstract}

DOI: 10.1103/PhysRevE.98.022128

\section{INTRODUCTION}

In the present paper we propose a rather general but simple model for a local search. In contrast to a global search, a local search is known to be concerned with the neighborhood of a given spot [1], called home. Such locally searching devices, living objects, agents, etc., return permanently to the home. In fact, they form a bound state with the home without real acting attractive forces. The behavior of maintaining a reference location and returning to it is called homing [2]. If the position of the home and the angle towards the home are known, then the method is called path integration [2-5].

The homing behavior might be based on idiothetic (internal) or allothetic (external) cues [6]. An external cue might be, for example, the position of the sun, odors, pheromones, or regional landmarks, while idiothetic cues are based on some internal storage mechanism $[7,8]$.

Such homing behavior is also known from insects such as ants, bees, and flies [5,9-12]. The home can be a nest or a source of food.

Various objects perform homing in different ways. The ant Cataglyphis builds a tortuous trajectory until a source of food is found and then goes back home in a straight path, indicating it has a rather accurate sense of the nest position [9-11]. In contrast, the fruit fly Drosophila melanogaster performs idiothetic homing after a food source is found. The fly explores the vicinity of the food, returns, and starts exploring again. This oscillating behavior is believed to fulfill the purpose of foraging, so the flies are then able to search for more food while keeping track of already found sources [12]. In all the mentioned examples of living objects, the trajectories always appear stochastic.

Furthermore, a new age of exploration has started to develop, from a technological point of view [13]. Explorer robots are being projected for missions in the ocean [14-16] and space [13,17]. Autonomous vehicles [16,18-20] will be used for data collection and local searches. In the development of a scientific understanding of these technologies and their devices, research in this field relates in many cases successfully to data and/or numeric models developed for animal navigation [13]. Often such devices are inspired by research on biological objects fulfilling different purposes, for example, a local search as a permanent search and return process. A better theoretic-mathematical understanding of local searches might also explain the performance of self-navigating objects.

In this paper we introduce a class of minimal stochastic models for local search that does not distinguish between the search and the return. Both epochs will follow the same law. The particular dynamic model and its simplicity will be 
justified by the allowance for an analytical tractability and that the model reproduces qualitatively several experimental findings. We use a Langevin equation that considers an active Brownian particle with constant speed whose spatial motion is two dimensional. The constant speed is common in a variety of models [21,22] and also has been observed in [5]. The model aims to mimic the motion of simple organisms; in particular, we focus the discussion of the analytic and numeric results of our model on findings for the fruit flies considered in [5].

We implement the local search around the home via a nonlinear coupling term between the heading angle of the particle and the angle formed by its current position and the home. Both angles effectively interact in a sequence of an escape and pursuit dynamics. To perform this dynamics, we assume that the agent possesses an internal storage mechanism for the current angles of the heading direction and of the position towards the home. The resulting spatial motion allows the particle to explore the vicinity of the home in order to find new food sources and searchers will consecutively return to the home.

In Sec. II we briefly introduce the model and then discuss in Sec. III the deterministic trajectories, which turn out to be a conservative system with an integral of motion. We continue in Sec. IV the discussion with noise present. The noise will be added to the angular dynamics. In many stochastic models Gaussian white noise is applied. However, we model the noise as a symmetric $\alpha$-stable white-noise source which also includes the case of a Gaussian noise with the particular choice $\alpha=2$. Thus, we are able to include different turning statistics of the heading angle including non-Gaussian white noise as it was observed in the searching motion of fruit flies and for other insects [23]. For a global search the Lévy flight hypothesis [1] is popular. However, for a local search, step lengths distributed according to a power law seems counterproductive. We do not know about models for local searchers with corresponding noise sources.

The noise serves as an uncertainty in the heading direction for the active particle. This uncertainty might have its origin in a limited capability to choose an exact direction of motion, due to external influences or the mechanics of the brain. Another source of randomness is the lack of information. Under the circumstance where it is unclear what the optimal heading direction is, the actual choice can become random. Those uncertainties cause in our model a steady-state distribution (Sec. IV B) of the particles centered at the home with exponentially decaying probability density at large distances. In Sec. V we discuss the local search characteristics and in particular we find an optimal noise strength for discovering food. In Sec. VI we generalize the model by allowing the coupling to the home to be dependent on the distance to the home. Finally, we summarize our findings in Sec. VII.

Several technical aspects concerning the $\alpha$-stable noise have been included in Appendixes A and B. They have been of importance for the investigations on stochastic dynamics driven by $\alpha$-stable noise in the angular dynamics. In Appendix A we give the foundation for the noise-dependent relaxation time of the system. It serves as the characteristic time for relaxation of angular asymmetry. For larger times, the system is approximated by an overdamped description as elucidated in Appendix B for various sources of $\alpha$ noise in the angular (a)

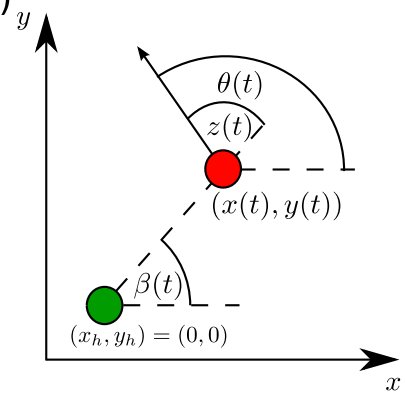

(b)

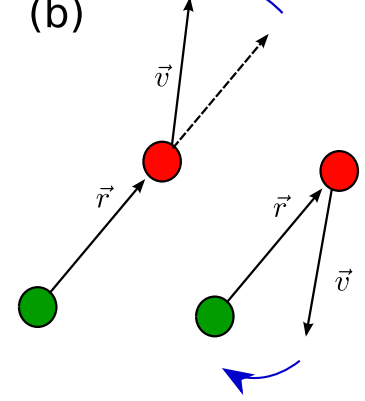

FIG. 1. (a) Schematic representation of coordinates. The angle $\theta(t)$ defines the current heading direction pointing along the actual velocity. The angle $\beta(t)$ is the direction of the vector from the home to the agent positioned at $\vec{r}(t)$. For convenience, the home is situated at the origin $\vec{r}_{h}=(0,0)$. (b) Sketch of the interaction between position and heading vectors. If the heading vector points outward from the home as the position vector always does, the heading vector becomes repelled from the position vector. In contrast, pointing homeward, the heading vector is attracted by the position vector.

dynamics. Appendix $\mathrm{C}$ describes the deterministic dynamics as a celestial mechanics with constant speed.

\section{MODEL}

We consider an active particle whose position vector is given by $\vec{r}(t)=\{x(t), y(t)\}$. We model a particle with constant speed $v_{0}$,

$$
\dot{\vec{r}}=\vec{v}(t)=v_{0}\left(\begin{array}{c}
\cos \theta(t) \\
\sin \theta(t)
\end{array}\right),
$$

where $\dot{\vec{r}}$ denotes the temporal derivative of the position vector $\vec{r}(t)$ and $\theta(t)$ is the heading angle of the particle, as depicted in Fig. 1.

As we choose the home to be situated at the origin of the Cartesian reference frame, the position vector $\vec{r}(t)$ points always out of the home in the direction of the particle's current position. The corresponding angle $\beta(t) \in[0,2 \pi)$ is given by

$$
\beta(t)=\arctan \frac{y(t)}{x(t)},
$$

as also sketched in Fig. 1. The time evolution of $\beta$ is determined by Eq. (1). For particles which arrive at times $t_{h}$ at the home $\vec{r}\left(t_{h}\right)=\vec{r}_{h}$, the angle $\beta\left(t_{h}\right)$ remains undefined. In these rare cases we will agree that the angle $\beta$ converges with the heading direction, i.e., $\beta\left(t_{h}\right)=\theta\left(t_{h}\right)$ holds. This choice corresponds to a particle that leaves the home in the radial direction. Notice that during the passage of the home, the angle $\beta$ jumps by $\pi$.

The search and return dynamics of the active particle is encoded in the evolution of the heading angle $\theta \in[0,2 \pi)$. The evolution of the heading direction contains the actual decision process of the searcher by selecting the future direction of its velocity. We assume that the heading evolves in time according to

$$
\dot{\theta}=\kappa \sin (\theta-\beta)+\frac{\sigma}{v_{0}} \xi(t) .
$$

While Eq. (1) is simply the mechanics of the motion with constant speed, Eq. (3) expresses the searcher's wish (i) 
to explore new space around the home and (ii) to return sequentially towards the home. It is the process for which the searcher needs the knowledge of the two angles $\beta(t)$ and $\theta(t)$. With the help of both, the searcher performs path integration.

The first term on the right-hand side (rhs), the deterministic term, can be motivated by an effective escape and pursuit dynamics, as it was also discussed in [24]. For a positive value of $\kappa$, the heading angle escapes the unstable outward direction $\theta(t)=\beta(t)$ and pursues the homeward direction $\theta(t)=\beta(t)+\pi$, which is to be stable. Such behavior is schematically indicated in Fig. 1(b). If the projection of the heading onto the position vector is positive, the heading becomes repelled from the position vector. In contrast, in the case in which the projection of the heading vector is negative or it is antiparallel to the position vector, the heading is attracted by the homeward direction.

The second term on the rhs of Eq. (3) $\xi(t)$ will be a symmetric $\alpha$-stable white-noise source. It serves as an uncertainty in the heading direction, caused by a decision process, by a limited knowledge of the heading direction or by external influences. We also point out that lack of information is a noise source. Lack of information can imply that a decision whether to turn left or right or move straight forward is a random choice, as it is unclear what would be the "right" choice. The noise strength is $\sigma$. The $\alpha$-stable white noise with $\alpha<2$ in the angular dynamics yields a continuous description for a run-and-tumble-like motion with a fast tumbling epoch [25], as it was also found and reported in an experimental study [5]. In the case of $\alpha=2$, increments of the angle are uncorrelated in time and with Gaussian support.

The equations of motion (1) and (3) take in polar coordinates $r(t)=\sqrt{x^{2}(t)+y^{2}(t)}$ and $\beta(t)$ given by (2) an especially simple form

$$
\dot{r}=v_{0} \cos (\theta-\beta)
$$

for the radial velocity and

$$
\dot{\beta}=\frac{v_{0}}{r} \sin (\theta-\beta)
$$

for the tangential velocity. Although the distance $r(t)$ and the position angle $\beta(t)$ are stochastic values, their respective equations of motion do not contain noise sources, as the Cartesian velocities also do not contain noise sources. Despite the stochastic character, the speed of the particle is always constant, i.e., $\dot{r}^{2}+(r \dot{\beta})^{2}=v_{0}^{2}$.

Defining now the angle $z(t) \in(-\pi, \pi]$ as the difference of the heading and the position angle, i.e., $z(t)=\theta(t)-\beta(t)$, we derive

$$
\begin{aligned}
\dot{r} & =v_{0} \cos (z), \\
\dot{\beta} & =\frac{v_{0}}{r} \sin (z),
\end{aligned}
$$

and consequently

$$
\dot{z}=\dot{\theta}-\dot{\beta}=\left(\kappa-\frac{v_{0}}{r}\right) \sin (z)+\frac{\sigma}{v_{0}} \xi(t) .
$$

These three equations are the stochastic nonlinear dynamics of the local searcher in polar coordinates. One immediately notices that the $\beta(t)$ dynamics (7) separates from the stochastic motion on the $(r, z)$ plane. The solutions of (6) and (8) determine, after insertion in (7) and its integration, the further behavior of the angle $\beta(t)$.

We also point out that in Eq. (8) a length scale $r_{c}$ has emerged for the first time in the description. It reads

$$
r_{c}=v_{0} / \kappa
$$

and defines the relative angular speed between the position vector and the heading vector. In the noise-free setting $(\sigma=0)$ both vectors rotate always either clockwise or counterclockwise. However, for distances smaller $r_{c}$ the heading rotates slower than the position vector. Otherwise the heading is faster. The search and return motion will be an oscillatory sequence around $r_{c}$ defined by the interplay of the two vectors.

\section{DETERMINISTIC MODEL}

We will now discuss the deterministic motion of our searcher. Without noise the time evolution (8) of the angle $z$ becomes

$$
\dot{z}=\left(\kappa-\frac{v_{0}}{r}\right) \sin (z)
$$

Equations (6), (7), and (10) define the dynamics of our deterministic searcher and have to be supplemented by initial conditions $r_{0}=r(t=0), z_{0}=z(t=0)$, and $\beta_{0}=\beta(t=0)$.

\section{A. Fixed points and separatrices}

The simplest solution in the $(r, z)$ plane is stationary fixed points at $z_{0}= \pm \pi / 2$ and $r_{0}=r_{c}$, with $\dot{r}=\dot{z}=0$. These fixed points are of the center type and have purely imaginary eigenvalues $\lambda$. In detail, these eigenvalues are equal for both centers $\lambda= \pm \mathrm{i} \kappa$, with $\kappa$ being the coupling strength from Eq. (3). The value $\kappa$ yields also the circulating frequency of the $\beta(t)$ dynamics in the $(x, y)$ plane. It is dependent on the initial sign of $z_{0}$ either clockwise or counterclockwise. This solution corresponds to a circular trajectory in the $(x, y)$ plane with radius $r_{0}$. The initial heading is perpendicular to the position vector and it remains so further on.

The second class of trivial solutions belongs to states $z=0, \pi$. Since $z(t)$ is periodic in $2 \pi$, the latter state is also equivalent to $z=-\pi$. States with $z(t)=0$ define the radially unbounded case. At arbitrary distances $r$ the particle moves radially away since $\theta(t)=\beta(t)$. In the noise-free situation solutions with $z=0$ never change since $\dot{z}=0$. So the trajectories become straight lines and the distance to the home grows unbounded. The particles escape with velocity $v_{0}$ to infinity

$$
\begin{aligned}
& r(t)=v_{0} t+r_{0}, \\
& \beta(t)=\beta_{0}, \\
& z(t)=0 .
\end{aligned}
$$

Deterministically, the state $z=0$ can be approached only if either $z_{0}=0$ or $z_{0}=\pi$. In the latter case the particle started with $z=\pi$ at $r_{0}>0$ will radially approach the home $z=\pi$ and pass it while the angle $z$ jumps to $z=0$, as the position angle jumps $\beta=\beta_{0}+\pi$ and then moves away along with $z=0$.

The distance $r=0$ possesses the meaning of a repelling boundary. It can be approached by $z=\pi$. The state at $(r=$ 

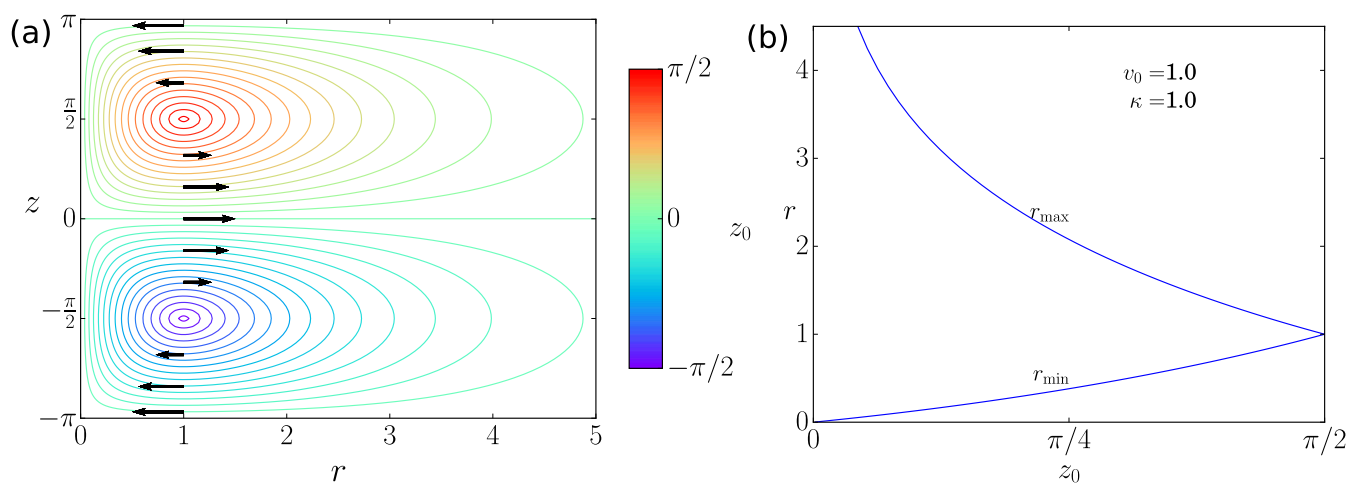

FIG. 2. (a) Sample trajectories in the $(r, z)$ plane according to Eq. (13). The color bar corresponds to the initial starting angle $z_{0}$ and thus colored trajectories belong also to different values of $X\left(z_{0}\right)$. (b) Minimal and maximal distances dependent on the initial angle $z_{0}$ for $r_{0}=r_{c}$. The parameters are $v_{0}=1$ and $\kappa=1$.

$0, z=\pi)$ is immediately left and the angle $z$ flips to $z=0$. This dynamic behavior is in agreement with the trajectories running near this boundary which are unable to attach to the boundary. Oppositely, the $z(t)$ dynamics becomes unlimited fast near $r=$ 0 as it follows from (10) due to the item proportional to $1 / r$ on the rhs. In consequence, the state $r=0$ plays the role of an extended saddle point with an incoming separatrix at $z=\pi$ and an outgoing one along $z=0$. This is also confirmed by the fact that the two lines play the role of a separatrix. Thus, both lines divide the $(r, z)$ space into two half planes on which either a clockwise or an anticlockwise periodic motion takes place. Both sets of solutions never merge.

\section{B. Oscillatory $(r, z)$ dynamics}

Other solutions correspond to bounded trajectories in the $(r, z)$ space and possess a maximal and a minimal distance from the home. The motion becomes a repetitive change of attraction and repulsion towards the home-pointing direction that defines an oscillatory motion in the $(r, z)$ plane.

Figure 2 presents the flow diagram of the $(r, z)$ dynamics. It is obtained when eliminating the time dependence by differentiating the angle $z$ with respect to the position $r$ as

$$
\frac{d z}{d r}=\frac{\dot{z}}{\dot{r}}=-\left(\frac{1}{r}-\frac{1}{r_{c}}\right) \tan (z) .
$$

Since distance $r$ and angle $z$ can be factorized in the differential equation (12), we can integrate and find parametrically $z(r)$. With the initial conditions $r_{0}$ and $z_{0}$, we can formally write the angle dependent on the position as

$$
\begin{aligned}
\sin [z(r)] \exp \left(-\frac{r}{r_{c}}\right) r & =X\left(z_{0}, r_{0}\right)=\sin \left(z_{0}\right) \exp \left(-\frac{r_{0}}{r_{c}}\right) r_{0} \\
& =\text { const. }
\end{aligned}
$$

This expression defines the formal solution $z(r)$. The parameter $X$ is an integral of motion for the $(r, z)$ dynamics. Therefore, the $(r, z)$ dynamics is conservative. The value of $X$ depends on the initial coordinates only. In particular, the sign of the initial angle also fixes the sign of $z(t)$ during the motion.

In the $(r, z)$ plane, the solutions are periodic, so we can choose the initial condition $r_{0}=v_{0} / \kappa=r_{c}$, as every trajectory at least crosses $r=r_{c}$. Hence, the integral of motion becomes a function of $z_{0}$, only, i.e., $X=X\left(z_{0}\right)$. This angle $z_{0}$ can be restricted to $z_{0} \in[-\pi / 2, \pi / 2]$. The motion along $z_{0}=0$ corresponds to $X=0$. The fixed points have $z_{0}= \pm \pi / 2$ with the $X$ value according to (13).

Figure 2(a) shows solutions of (13) in the $(r, z)$ plane, with the parameters $v_{0}=1, \kappa=1$, and $r_{0}=r_{c}$. The initial angle $z_{0}$ is varied corresponding to the color bar. As already outlined, there is no trajectory which crosses the separatrices $z=0$ or $z= \pm \pi$; in fact, the angle $z(t)$ is bounded when considering a specific trajectory. This means that the angular momentum

$$
L=r^{2} \dot{\beta}=v_{0} r \sin (z)=v_{0} \exp \left(\frac{r}{r_{c}}\right) X\left(z_{0}\right)
$$

along a trajectory never changes its sign. It follows that in the deterministic model the particles either move with $z>0$ in a clockwise manner or with $z<0$ in a counterclockwise fashion around the home in the Cartesian coordinate system. The upper and lower half planes in Fig. 2(a) correspond to particles starting with either $z_{0}>0$ or $z_{0}<0$, respectively.

Trajectories in the $(r, z)$ plane approach the maximal $r_{\max }$ and minimal $r_{\min }$ distances (perihelion and aphelion) if $d r / d z$ vanishes. To formulate a criterion for these positions and following Eq. (12) we set $z= \pm \pi / 2$ in Eq. (13) and obtain for both distances

$$
r_{\text {max }, \min } \exp \left(-\frac{\kappa}{v_{0}} r_{\max , \min }\right)=\left|X\left(z_{0}\right)\right| .
$$

The extremal distances dependent on the initial angle $z_{0}$ for $r_{0}=r_{c}$ are plotted in Fig. 2(b) for the parameters $v_{0}=1$ and $\kappa=1$. At the extremal distances the radial velocity $\dot{r}$ vanishes. This can be seen when considering the kinetic energy of the constant speed particle, given by $E_{\text {kin }}=\left(\dot{x}^{2}+\dot{y}^{2}\right) / 2=v_{0}^{2} / 2$. In polar coordinates the energy reads $E_{\text {kin }}=\left(\dot{r}^{2}+r^{2} \dot{\beta}^{2}\right) / 2$. We express the energy as

$$
E_{\mathrm{kin}}=\frac{1}{2} \dot{r}^{2}+\frac{1}{2} v_{0}^{2} \sin ^{2}[z(r)]=\frac{1}{2} v_{0}^{2}
$$

through (7). For the radial velocity it follows that

$$
\dot{r}^{2}=v_{0}^{2}\left\{1-\sin ^{2}[z(r)]\right\} .
$$

At $r_{\text {max } \min }$ the radial velocity vanishes $\dot{r}\left(r=r_{\max , \min }\right)=0$. Those are turning points; the motion away from the home turns to motion towards the home and vice versa. The corresponding tangential velocity is maximal $\dot{\beta}\left(r=r_{\max , \min }\right)=$ 

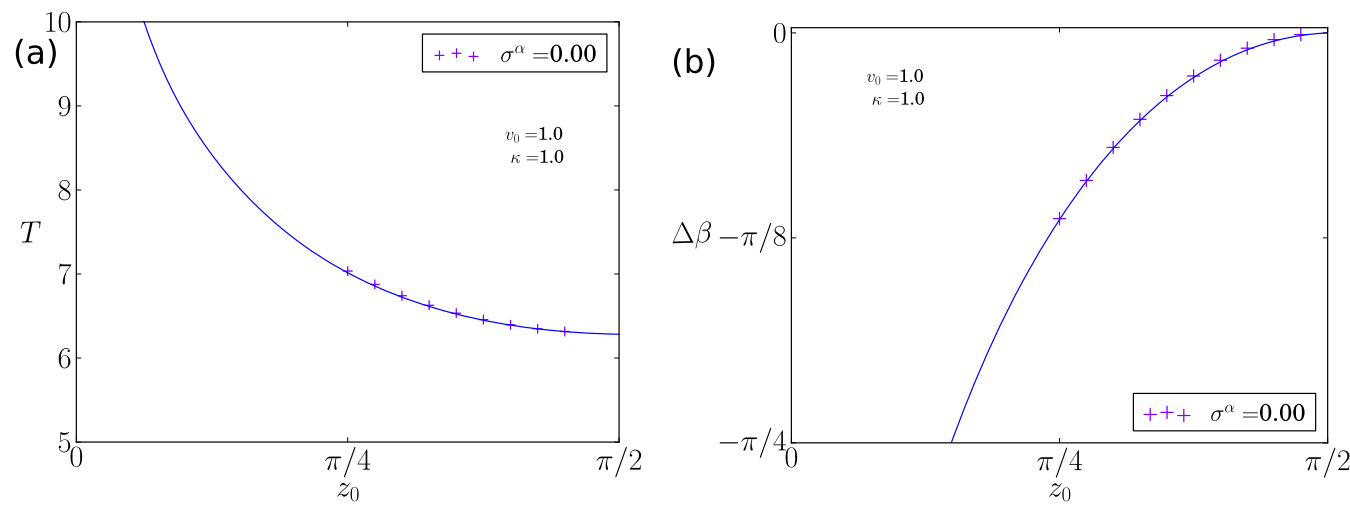

FIG. 3. (a) Period of one cycle dependent on the initial angle $z_{0}$. The solid line corresponds to Eq. (18). Symbols are from simulations of Eqs. (1) and (3) with vanishing noise $(\sigma=0)$. (b) Value of the apsidal precession of the orbit during one revolution as a line corresponding to Eq. (20). Symbols are from simulations of Eqs. (1) and (3) with vanishing noise.

$\pm v_{0}$. At $r=r_{c}$ the radial velocity is maximal, as $0=d \dot{r} / d r=$ $v_{0} \sin (z) \tan (z)\left(1 / r-1 / r_{c}\right)$ is solved by $r_{c}$. The maximal radial velocity is given by $\dot{r}\left(r=r_{c}\right)= \pm v_{0} \sqrt{1-\sin ^{2}\left(z_{0}\right)}$, under the condition that we set $r_{0}=v_{0} / \kappa$. The tangential velocity is therefore given by $\dot{\beta}\left(r=r_{c}\right)=v_{0} \sin \left(z_{0}\right)$.

\section{Period length and shift of the position angle}

As the $(r, z)$ dynamics is periodic, we can determine a period length. The period $T$ of one cycle can be calculated from Eq. (17). The angle can be eliminated using Eq. (13) and we find for the period the expression

$$
T\left(z_{0}\right)=\frac{2}{v_{0}} \int_{r_{\min }}^{r_{\max }} d r \frac{1}{\sqrt{1-\left(\frac{X\left(z_{0}\right)}{r}\right)^{2} \exp \left(\frac{2 r}{r_{c}}\right)}} .
$$

Figure 3 presents as a solid line the numerical evaluation of the periods dependent on the absolute value of the angle $\left|z_{0}\right|$ according to Eq. (18). Simulation results of the deterministic version of Eqs. (1) and (3) are shown with pluses. The value decays monotonically for growing $z_{0}$ performing smaller excursions starting with the escaping trajectory for $z_{0}$ until it moves along the circular orbit at the stationary center $\left|z_{0}\right|=$ $\pi / 2$.

Up to now, we have considered only the reduced $(r, z)$ dynamics. As our particles are moving in the $(x, y)$ plane, we determine now the value of the position angle $\beta$ dependent on the distance $r$. Using the equation for the tangential velocity (5) and Eq. (13), we write the differential for the angle $\beta$ dependent on the radial position $r$ as $d \beta / d r$ and integrate formally with initial distance $r_{0}$ and angle $\beta_{0}$ :

$$
\beta(r)=\beta_{0}+\int_{r_{0}}^{r} \frac{d r}{r} \frac{1}{\sqrt{\left(\frac{r}{X\left(z_{0}\right)}\right)^{2} \exp \left(-2 \frac{r}{r_{c}}\right)-1}} .
$$

After one cycle the trajectory has moved through the maximal and the minimal distance $r_{\text {max,min }}$, reaches again $r_{0}$, and has accumulated an angular shift $\Delta \beta$ of

$$
\Delta \beta\left(z_{0}\right)=-2 \pi+2 \int_{r_{\min }}^{r_{\max }} \frac{d r}{r} \frac{1}{\sqrt{\left(\frac{r}{X\left(z_{0}\right)}\right)^{2} \exp \left(-2 \frac{r}{r_{c}}\right)-1}} .
$$

Values of the shift at the extremal elongations dependent on the initial angle difference $z_{0}$ are presented in Fig. 3. The shift according to Eq. (20) is shown as a line, while the pluses correspond to the deterministic case of the system according to Eqs. (1) and (3).

\section{Dynamics in the $(x, y)$ plane}

The motion in the $(x, y)$ space is reminiscent of the apsidal precession of the planetary motion, where the aphelion and perihelion shift during one revolution around the center of gravity. We elaborate on this analogy in Appendix C. The shape of the trajectories is reminiscent of Lissajous curves, or rose curves, and the motion is in general quasiperiodic. An interesting value is the time $T_{\text {ros }}$ needed for the precession to rotate more than $2 \pi$, meaning that a rosette has completely formed. One can estimate this time after determining the number of leaves $K\left(z_{0}\right)$ necessary for the completion of the rosette. It reads $K\left(z_{0}\right)=2 \pi / \Delta \beta\left(z_{0}\right)$. Therefore, it becomes

$$
T_{\text {ros }}\left(z_{0}\right)=\frac{2 \pi}{\Delta \beta\left(z_{0}\right)} T\left(z_{0}\right)
$$

with expressions from (18) and (20).

Finally, we can express the bounded trajectories in the $(x(r), y(r))$ plane through the parameter $r$,

$$
\begin{aligned}
& x(r)= \begin{cases}r_{c} \cos (\beta), \quad z_{0}= \pm \pi / 2, r_{0}=r_{c} & \forall \beta \in[0,2 \pi) \\
r \cos [\beta(r)+l \Delta \beta] & \text { otherwise, }\end{cases} \\
& y(r)= \begin{cases}r_{c} \sin (\beta), \quad z_{0}= \pm \pi / 2, r_{0}=r_{c} & \forall \beta \in[0,2 \pi) \\
r \sin [\beta(r)+l \Delta \beta] & \text { otherwise, }\end{cases}
\end{aligned}
$$

where $\beta(r)$ is due to (20), with $r \in\left[r_{\min }, r_{\max }\right]$, and the positive number of revolutions around the home $l \in \mathrm{N}^{+}$. The first case is the circular trajectory. If the home $\left(x_{h}, y_{h}\right)$ is not situated at the origin of the coordinate system one can shift $x^{\prime}(r)=x(r)-x_{h}$ and $y^{\prime}(r)=y(r)-y_{h}$.

Figure 4 shows sample trajectories according to Eqs. (22) and (23), with $r_{0}=r_{c}, v_{0}=1$, and $\kappa=1$. The location of the 

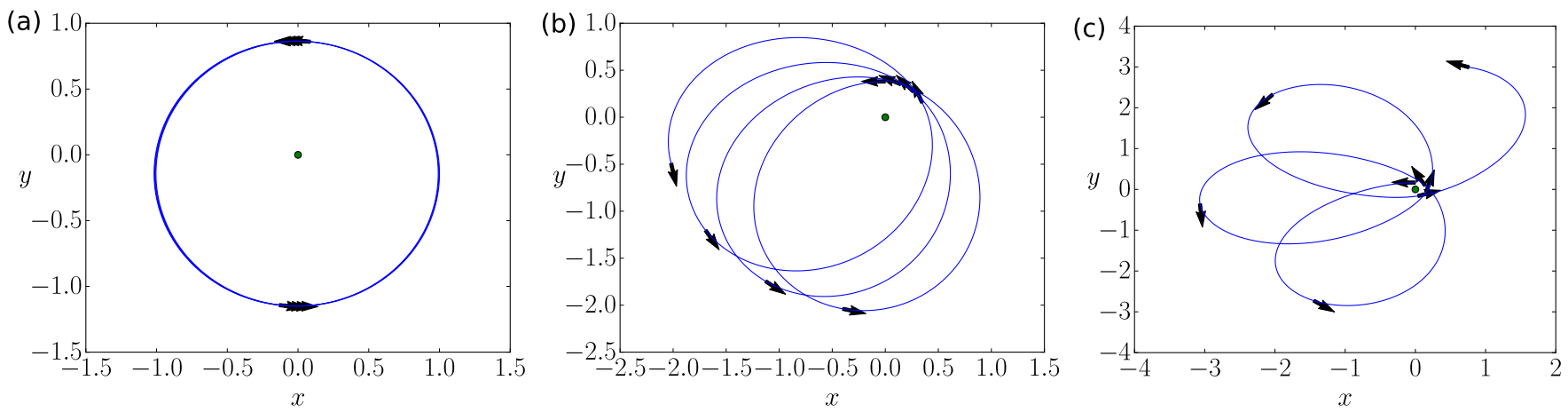

FIG. 4. Sample trajectories in the $(x, y)$ plane. The green dot marks the home. The particles for all trajectories start at the minimal distance from the home $x_{0}=0, y_{0}=r_{\min }\left(z_{0}\right)$, and the trajectories are plotted until the maximal distance is reached the fourth time. Shown are three different initial $z_{0}$. From left to right, $z_{0}=\pi / 2.2, z_{0}=\pi / 4$, and $z_{0}=\pi / 8$. The arrows indicate the direction of motion. The inverse motion is also possible. The parameters are $v_{0}=1, \kappa=1$, and $r_{0}=1$.

home is marked with a green dot. The pictures correspond to three different initial angles $z_{0}$, i.e., $z_{0}=\pi / 2.2$ [Fig. 4(a)], $z_{0}=\pi / 4$ [Fig. 4(b)], and $z_{0}=\pi / 8$ [Fig. 4(c)]. The initial position in the $(x, y)$ plane is chosen as $x_{0}=0$ and $y_{0}=$ $r_{\min }\left(t_{0}\right)$. The arrows indicate the direction of motion for these specific initial conditions. While for $z_{0}=\pi / 2.2$ the trajectory is almost circular, it becomes more and more stretched the smaller $z_{0}$ becomes, the maximal distance increases, and the minimal distance gets closer to zero.

\section{STOCHASTIC MODEL}

We will now return to the system with noise. The equations of motion are given by (6)-(8). Compared to the deterministic case, one has now two more parameters: (i) the parameter $\alpha$ for the noise type and (ii) the parameter $\sigma$, the noise strength. It will be of importance that the noise acts only on the $\theta(t)$ dynamics, and the respective $z(t)$ dynamics, of the motion and that the radial dynamics is perpendicular to the $z$ dynamics. The speed of the particle is always constant.

At first one finds that an unbounded motion becomes unlikely in the stochastic system. Trajectories remain with high probability at finite distances from the home. Therefore, generally the noise stabilizes the motion of the searcher.

\section{A. Stochastic dynamics}

Only the noise type controls jumps of the heading angle. This behavior is illustrated in Fig. 5. We show sample trajectories in the $(x, y)$ plane for two different noise sources: Gaussian white noise $\alpha=2.0$ [Fig. 5(a)] and $\alpha=0.5$ [Fig. 5(b)]. The noise strength is chosen such that the influence of the deterministic part of the time evolution of the heading (3) is still recognizable, $\sigma^{\alpha}=0.01$. The particle starts at the home, marked as a green dot at $(x, y)=(0,0)$, and a time frame of $\Delta t=50$ is shown. While the trajectory in Fig. 5(a) is wiggling, due to many small noise-induced changes in the direction of motion, the trajectory in Fig. 5(b) with small $\alpha=0.5$ is rather smooth, corresponding to almost no change in the deterministic heading and with one clear sudden jump at $(x, y)=(4,2)$.

Increasing the noise strength suppresses the deterministic influence on the trajectories, as can be seen in Fig. 6. The noise strength $\sigma^{\alpha}=0.5$ was changed compared with Fig. 5 . The particles again started at the home $(x, y)=(0,0)$ and moved for the same time interval $\Delta t=50$. The deterministic part of the motion is no longer visible and also the trajectory on the right $(\alpha=0.5)$ appears to be confined to a smaller region of space. While the trajectory seems to be confined to a small region, we show in Sec. IV B that the overall probability density function to find a particle at a specific point $(x, y)$ is independent of the noise type $\alpha$ and the noise strength $\sigma$.
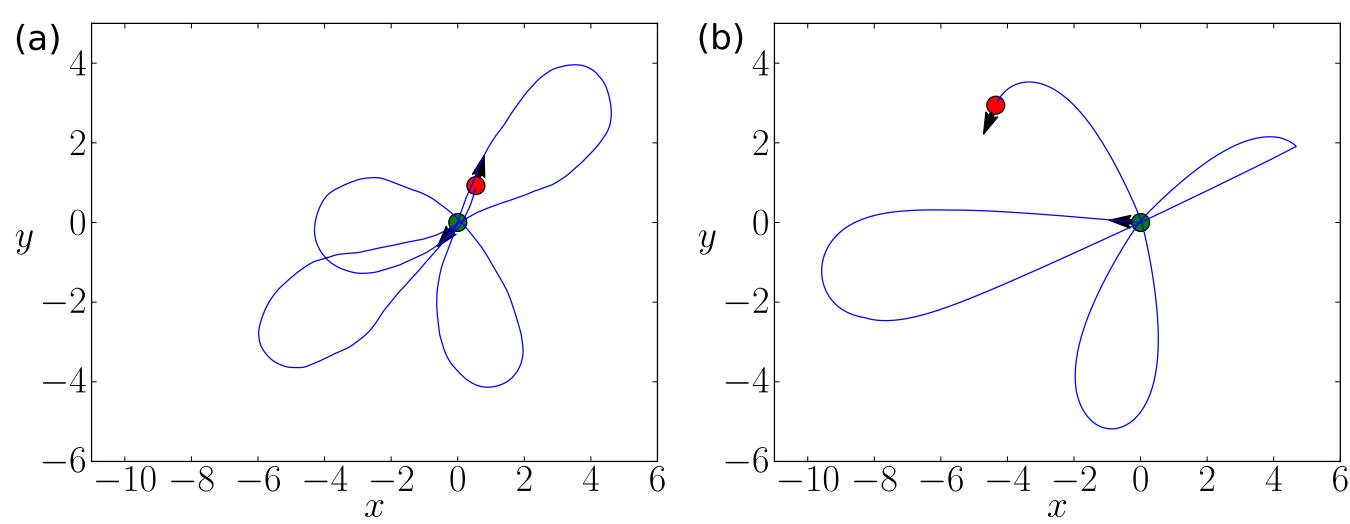

FIG. 5. Sample trajectories with low noise strength $\sigma^{\alpha}=0.01$ in the $(x, y)$ plane. The green dot marks the home. The particle starts at $(0,0)$ and moves for $\Delta t=50$ (red dot). The parameters are $v_{0}=1, \kappa=1$, and (a) $\alpha=2$ and (b) $\alpha=0.5$. 

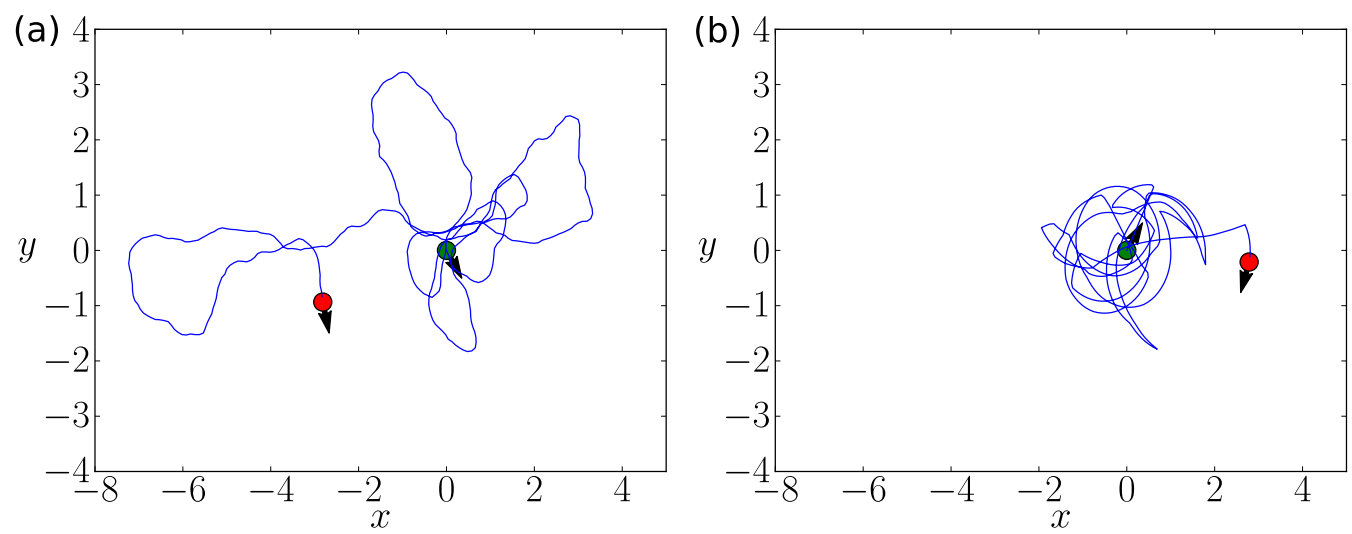

FIG. 6. Sample trajectories with high noise $\sigma^{\alpha}=0.5$ in the $(x, y)$ plane. The particle starts at $(0,0)$ (green dot) and moves for $\Delta t=50$ (red dot). The parameters are $v_{0}=1, \kappa=1$, and (a) $\alpha=2$ and (b) $\alpha=0.5$.

\section{B. Spatial distribution}

One measure to characterize the spatial extension of the search is the marginal density of the distance from the home. Here we will look for its asymptotic stationary expression as the result of solving the corresponding Fokker-Planck equation (FPE). To find this marginal density we inspect the probability density function (PDF) of the stochastic dynamics under consideration. It is the transition $\operatorname{PDF} P\left(r, z, \beta, t \mid r_{0}, z_{0}, \beta_{0}, t_{0}\right)$ that determines the density in three-dimensional space $(r, z, \beta)$ at time $t$, if started with the initial conditions $r_{0}, z_{0}$, and $\beta_{0}$ at $t_{0}$. Likewise in the deterministic case, we immediately realize that the stochastic $\beta$ dynamics separates from the two other variables $(r, z)$. It holds that $P(r, z, \beta, t)=P(r, z, t) P(\beta, t \mid r, z)$, where we have omitted the initial conditions for simplicity, meaning that the $(r, z)$ dynamics is independent of the $\beta$ dynamics. Therefore, to get the marginal spatial density, we may restrict consideration to the PDF $P\left(r, z, t \mid r_{0}, z_{0}, t_{0}\right)$ for finding a particle at distance $r$ and having the angle $z$ at time $t$ if started at time $t_{0}$ at distance $r_{0}$ with $z_{0}$.

Being interested in the asymptotic stationary limit when initial conditions are forgotten, we omit here and further on the initial conditions and use the notation of the PDF. Given Eqs. (6) and (10), we can write down the corresponding FokkerPlanck equation [26,27]

$$
\begin{aligned}
\frac{\partial}{\partial t} P(r, z, t)= & {\left[-v_{0} \frac{\partial}{\partial r} \cos (z)+\frac{\partial}{\partial z}\left(\frac{v_{0}}{r}-\kappa\right) \sin (z)\right.} \\
& \left.+\left(\frac{\sigma}{v_{0}}\right)^{\alpha} \frac{\partial^{\alpha}}{\partial|z|^{\alpha}}\right] P(r, z, t) .
\end{aligned}
$$

We assume a steady state $P(r, z, t \rightarrow \infty)=P_{0}(r, z)$, with $\partial P_{0}(r, z, t) / \partial t=0$, and make a separation ansatz

$$
P_{0}(r, z)=P_{0}(r \mid z) P_{0}(z) \text {. }
$$

Since the noise spreads the probability homogeneously around the angular dynamics and as no effective force repels the noisy shifts, no direction $z$ is preferred. Therefore, we assume that $z(t)$ becomes equidistributed after the relaxation time $\tau$ from Eq. (33) and we set $P_{0}(z)=1 / 2 \pi$. Further on, it turns out that this homogenization in the angular dynamics results also in an asymptotic spatial distribution independent of $z$, i.e., $P_{0}(r \mid z) \rightarrow P_{0}(r)$. Afterward, we find for the latter radial PDF the equation

$$
0=\left[-\frac{\partial}{\partial r}+\frac{1}{r}-\frac{1}{r_{c}}\right] v_{0} \cos (z) P_{0}(r),
$$

with $r_{c}$ from Eq. (9). The PDF no longer depends on the angle $z$, so we can drop the cosine function and integrate; the radial $\mathrm{PDF}$ is given by

$$
P_{0}(r)=\frac{r}{r_{c}^{2}} \exp \left(-\frac{r}{r_{c}}\right) .
$$

Including the angle $z$, we get

$$
P_{0}(r, z)=\frac{r}{2 \pi r_{c}^{2}} \exp \left(-\frac{r}{r_{c}}\right)
$$

Surprisingly, the spatial distribution, and therefore the probability to find a particle at a specific distance from the home, depends neither on the noise type nor on the noise strength. The reason behind this is that the noise acts perpendicular to the motion of particles. The distribution (27) is even valid and holds true for $\sigma=0$ if the system is initially distributed according to (27). The spatial distribution $P_{0}$ has a maximum at $r=r_{c}=v_{0} / \kappa$. Increasing the coupling strength $\kappa$ and keeping the speed $v_{0}$ fixed shifts the maximum closer to the home and the peak becomes more pronounced. The stronger the coupling towards the home, the closer to the home the maximum is situated. Increasing the speed $v_{0}$ leads to a growth of the distance between home and maximum and it broadens the spatial distribution $P_{0}(r)$.

Returning to Cartesian coordinates, the PDF reads

$$
P_{0}(x, y)=\frac{1}{2 \pi r_{c}^{2}} \exp \left(-\frac{1}{r_{c}} \sqrt{x^{2}+y^{2}}\right) .
$$

This PDF has maximal probability density to find the particle at the home $(0,0)$. We mention that the stationary PDF of the angle $\beta$ is also uniform at all distances.

Figure 7 shows the stationary radial PDF $P_{0}$ from (27) as a line in comparison with simulation results of Eqs. (1) and (3), with the position angle defined by (2). The simulations confirm that indeed the radial PDF is independent of noise type $\alpha$ and noise strength $\sigma$. We underline that the stationary spatial density qualitatively agrees with the experimental findings for the observed residence probability of a fruit fly as reported in 


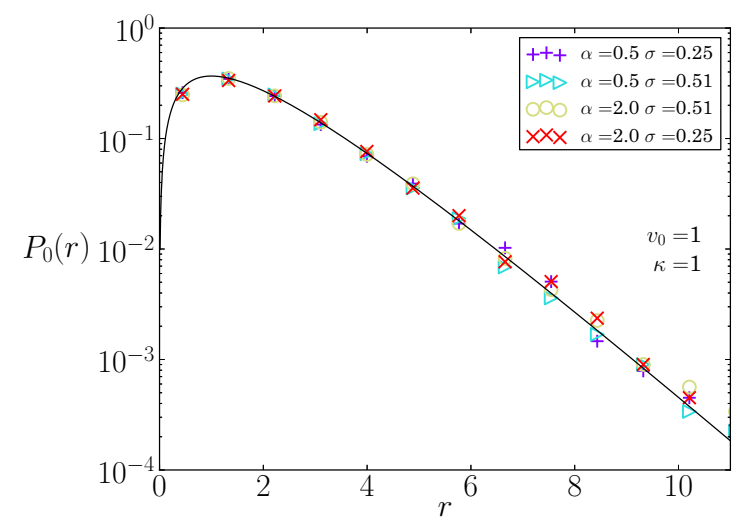

FIG. 7. Stationary radial PDF $P_{0}(r)$. The symbols are from simulations of Eqs. (1) and (3), with (2), and the black line corresponds to Eq. (27).

[5]. Taking the speed $v_{0}=10 \mathrm{~mm} / \mathrm{s}$ from [5] and judging from Fig. S4 in [5], we consider $\kappa=0.5 \mathrm{~s}^{-1}$ to be a realistic value for a fly.

\section{Relaxation time $\tau$}

In order to investigate how the stationary radial PDF is approached, it is useful to consider the integral of motion $X\left(z_{0}, r_{0}\right)$ from (13) together with the distance $r$ as a variable, as shown in Fig. 8. Deterministic trajectories with initial conditions $\left(r(t=0)=r_{0}, z(t=0)=z_{0}\right)$ become straight lines in the $(r, X)$ space, as $X$ is a constant, i.e., $\dot{X}=0$. Deterministic trajectories with different initial conditions $z_{0}$ and $r_{0}=r_{c}$ are shown in the $(r, X)$ space in Fig. 8 as color coded straight lines. The colors correspond to the respective trajectories of Fig. 2.

The envelope equals the unnormalized spatial distribution (27). The transformation from the $(r, z)$ plane to the $(r, X)$ plane does not preserve the direction of motion towards and away from the home; both happen on the same line.

In the noise-driven system the value of $X$ loses the meaning of an integral of motion. It becomes stochastic and, in consequence, time dependent. The noise allows for a vertical motion in Fig. 8. The black trajectories in Figs. 8(a) and 8(b) show the previous trajectories of Fig. 5 with low noise intensity $\sigma^{\alpha}=0.01$ and Figs. 8(c) and 8(d) show the trajectories of Fig. 6 with $\sigma^{\alpha}=0.5$. In Figs. 8(e) and 8(f) we show as a black line sample trajectories for $\sigma^{\alpha}=4.0$. In Figs. 8(a), 8(c), and
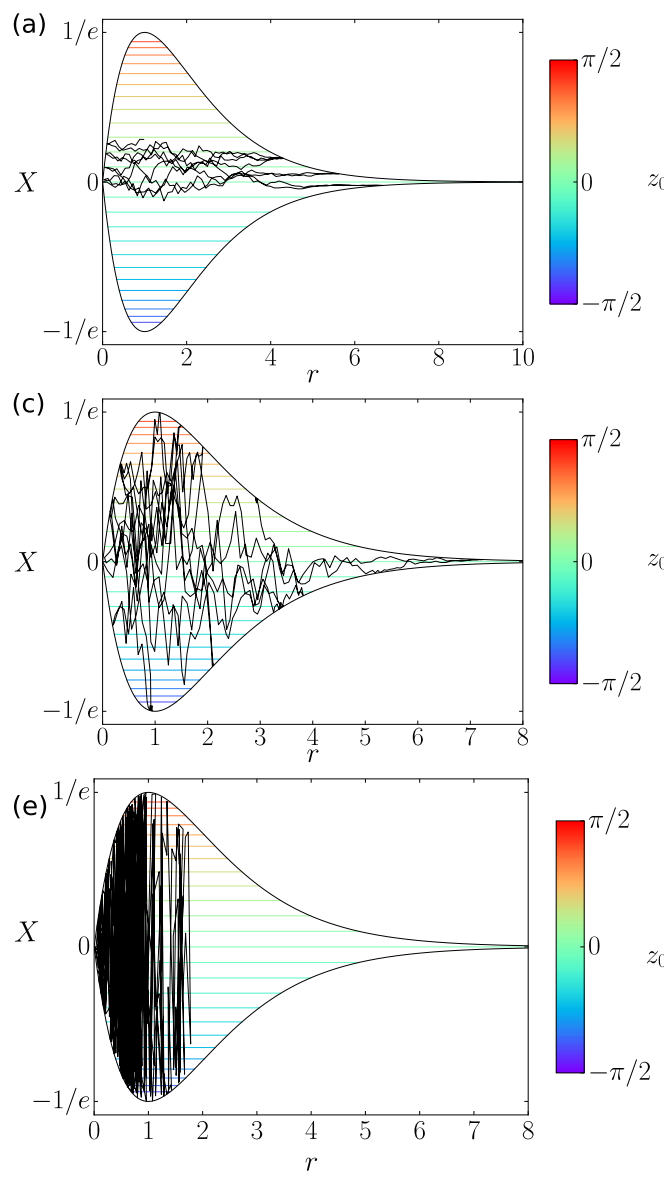
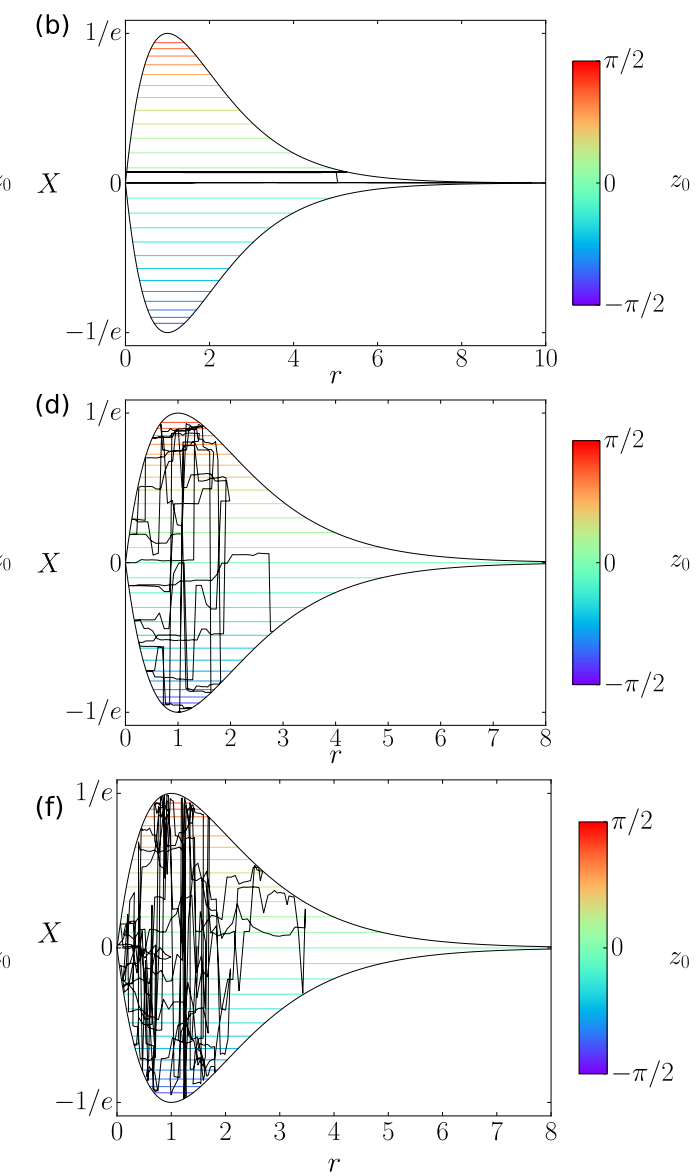

FIG. 8. (a) and (b) Trajectories of Fig. 5 with $\sigma^{\alpha}=0.01$ in the ( $\left.r, X\right)$ plane. (c) and (d) Trajectories of Fig. 6 with $\sigma^{\alpha}=0.5$ in the $(r, X)$. (e) and (f) Sample trajectories with high noise strength $\sigma^{\alpha}=4.0$. The total trajectory length is $t=50$, corresponding to (a) and (b) $t=0.5 \tau$, (c) and (d) $t=25 \tau$, and (e) and (f) $t=200 \tau$, with $\tau$ the relaxation time from (33). Color coded straight horizontal lines are various deterministic paths with constant $X\left(z_{0}, r_{0}=r_{c}\right)$ values. Colors correspond to the deterministic motion in the $(r, z)$ plane of Fig. 2. The parameters are $v_{0}=1$, $\kappa=1$, and (a), (c), and (e) $\alpha=2$ and (b), (d), and (f) $\alpha=0.5$. 

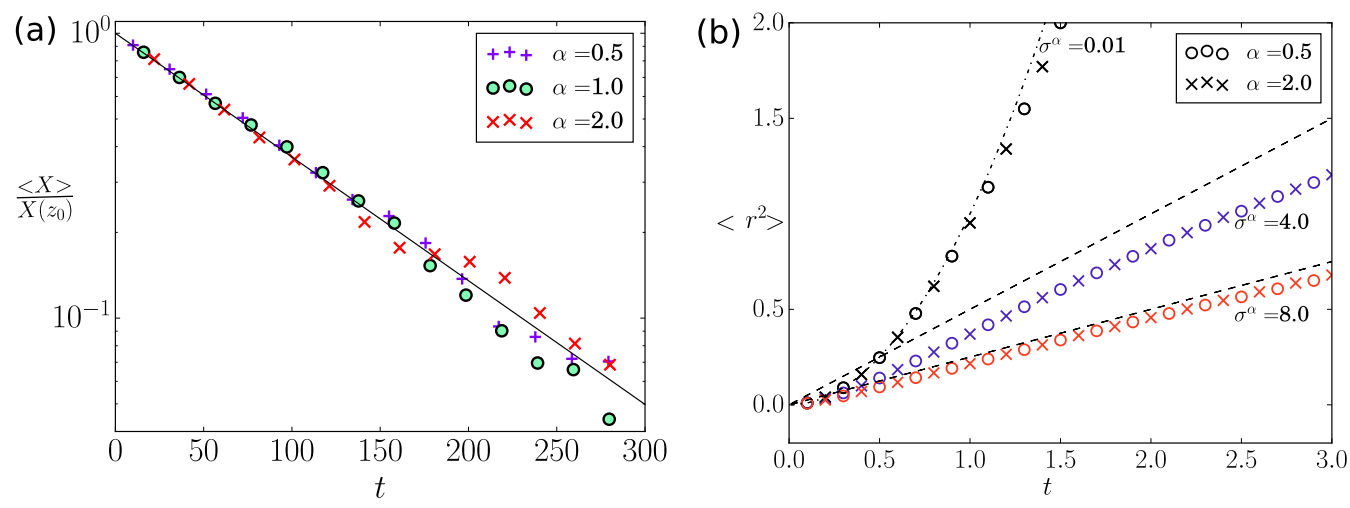

FIG. 9. (a) Symbols show the normalized ensemble average of $X$ obtained from simulation of (1) and (3), with $v_{0}=1, \kappa=1, \sigma^{\alpha}=0.01$ $(\tau=100)$, and initial $X(t=0)=X\left(z_{0}\right)$. The black line shows exponential decay according to (32). (b) Mean-square displacement $\left\langle r^{2}\right\rangle$ from simulations of particles started at $\left(x_{0}, y_{0}\right)=\left(r_{c}, 1\right)$ as at this distance the deterministic influence vanishes, with $r_{c}=1$, as $v_{0}=1$ and $\kappa=1$ for two different values of $\alpha$, each drawn for three different values of $\sigma^{\alpha}$. The dashed line corresponds to Eq. (36). The dash-dotted line corresponds to ballistic growth proportional to $t^{2}$. The plotted time frame is well below when the steady-state spatial distribution is reached.

8(e) the noise type corresponds to Gaussian white noise $\alpha=2$ and in Figs. 8(b), 8(d), and 8(f) to $\alpha=0.5$.

Three things should be noted when looking at the pictures. (i) The different noise types act differently: While Gaussian white noise causes rather continuous small vertical changes, the noise with $\alpha=0.5$ acts mostly through sudden jumps, as can be clearly seen in Figs. 8(a)-8(d). The trajectories for $\alpha=0.5$ stay a rather long time with a specific deterministic trajectory compared to the Gaussian noise case. (ii) The noise strength influences the time needed for a vertical motion of the particle, as can be seen following the pictures in a column. With increasing noise strength, the particles are distributed faster. (iii) In the radial direction, however, an increasing noise strength slows down the motion of the particle, which is particularly clearly visible in the Gaussian white-noise column. Interestingly, the relaxation time $\tau$ is equal for the two trajectories in each row, as we will show next, although their behavior appears quite different.

The vertical motion, i.e., the motion in the $X$ direction, can be characterized by the time evolution of the normalized ensemble average

$$
\langle X\rangle(t)=\int_{0}^{\infty} d r \int_{-\pi}^{\pi} d z X(r, z) P\left(r, z, t \mid r_{0}, z_{0}, t_{0}\right)
$$

conditioned to the initial values $r_{0}$ and $z_{0}$ at $t_{0}$ with the respective value $\langle X\rangle\left(t_{0}\right)=X_{0}=X\left(r_{0}, z_{0}\right)$ according to (13). This evolution is governed by the linear differential equation

$$
\frac{d}{d t}\langle X\rangle=-\frac{1}{\tau}\langle X\rangle
$$

A derivation of this equation based on the FPE multiplied by $X$ and integrated over $r$ and $z$ is given in the Appendix A. As result of (31) we obtain

$$
\langle X(t)\rangle=X_{0} \exp \left(-\frac{t}{\tau}\right)
$$

with

$$
\tau=\left(\frac{v_{0}}{\sigma}\right)^{\alpha}
$$

This timescale $\tau$ has the meaning of a relaxation time and is originated by the noise; without noise, no relaxation takes place since $\tau \propto 1 / \sigma^{\alpha} \rightarrow \infty$. With noise, any initial state $X_{0}$ is forgotten for $t \gg \tau$, as can be seen in Fig. 9(a). For three different values of $\alpha$ but with the same relaxation time $\tau$, the time-dependent averages $\langle X(t)\rangle$ obtained from simulation of Eqs. (1) and (3) with (2) are plotted together with Eq. (32) as a line. Hence, it is the time $\tau$ which is needed to vertically redistribute a trajectory in Fig. 8. We also remark that $\tau$ is the time after that a freely moving active particle with $\kappa=0$ has forgotten its initial heading direction [25].

Horizontal motion, the motion in the radial direction, in Fig. 8 is also governed by the noise-dependent timescale $\tau$ but by an effect acting oppositely as in case of the $X$. This means that with increasing $\tau$ the particles spread slower in the radial direction, while they spread faster in the $X$ direction, as was discussed above. We elucidate this effect by deriving an overdamped description of the nonlinear $(r, z)$ dynamics in Appendix B. We call it an overdamped description as the angle $z$ is the fast variable and the dynamics of the distance $r$ is assumed to be slow. The equation which determines then, for $t \gg \tau$, the evolution of the marginal radial PDF $P\left(r, t \mid r_{0}, t_{0}\right)$ is a Smoluchowski equation; all dependences on the various $\alpha$ values of the noise are expressed through the relaxation time $\tau$ as defined in (33). It becomes

$$
\frac{\partial}{\partial t} P=D_{\mathrm{eff}} \frac{\partial}{\partial r}\left[\frac{\partial}{\partial r} P-\left(\frac{1}{r}-\frac{\kappa}{v_{0}}\right) P\right],
$$

with the effective diffusion coefficient

$$
D_{\text {eff }}=\frac{v_{0}^{2} \tau}{2}
$$

The overdamped approximation is valid if the effective diffusion coefficient remains finite. The expression (35) of the latter coincides with results for freely moving active particles $\kappa=0$ with angular driving by $\alpha$-stable noise [25].

Note that the overdamped description is solved for the steady state by our previous solution (27). We also underline that the spatial relaxation proportional to the relaxation time, especially around $r \approx r_{c}$, follows the mean-square 

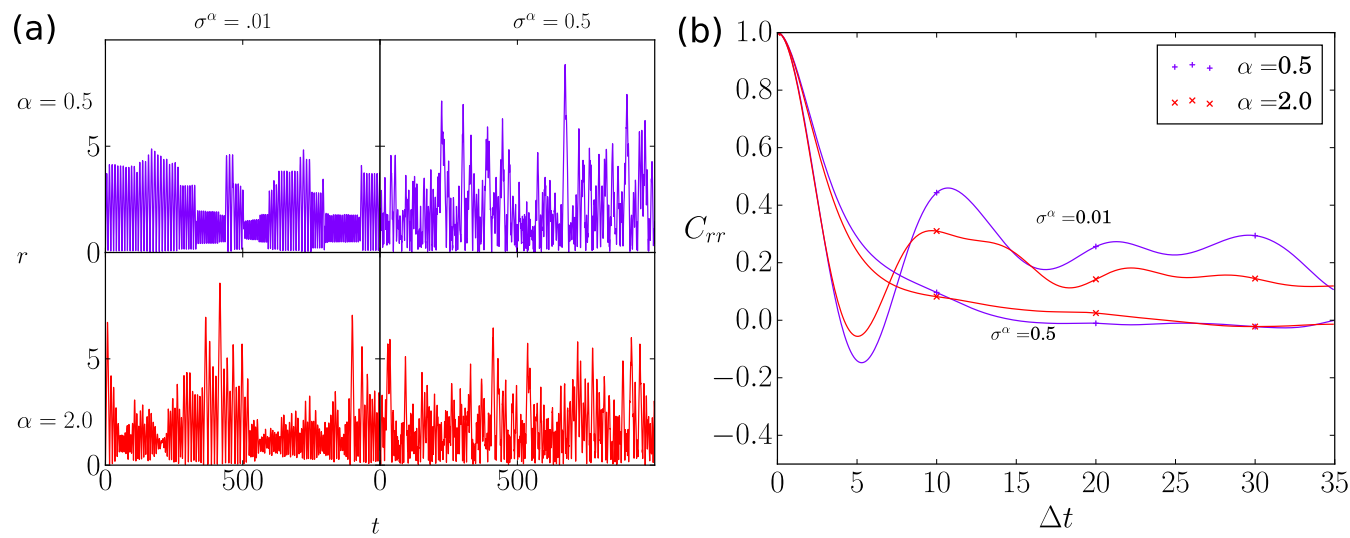

FIG. 10. (a) Distance $r(t)$ from the home dependent on time for two different noise types and two different noise strengths. (b) Corresponding autocorrelation function of distances $C_{r, r}(\Delta t)$. The values of $\alpha$ and $\sigma$ are given in the graphs. The other parameters are $v_{0}=1$ and $\kappa=1$.

displacement (MSD)

$$
\left\langle r^{2}\right\rangle \sim v_{0}^{2} \tau t
$$

Figure 9(b) displays as symbols the MSD obtained from simulations for particles started at $(x(t=0), y(t=0))=$ $\left(r_{c}, 0\right)$ and as a dashed line Eq. (36), with the noise strength and type as indicated in the figure. The displayed time frame is small compared to the timescale for the establishment of the steady state. With increasing noise strength, the simulation results for MSD align with (36). This implies that with increasing noise strength $\sigma$, the relaxation in the radial direction slows down. We also show the MSD for a low noise strength $\sigma^{\alpha}=0.01$. There the particle moves in the ballistic regime and the MSD grows proportionally to $v_{0}^{2} t^{2}$, as indicated by the dash-dotted line. The different dependence on the relaxation time of the radial and vertical motions in the $(r, X)$ plane will be the key element for the existence of an optimal time for a local search.

\section{LOCAL SEARCH}

The local search consists of a search and a return part. In our model both tasks are described by the same stochastic dynamics. We discuss now the return and afterward the search part.

\section{A. Sojourn time}

Our searcher performs a quasiperiodic motion around the home. Figure 10(a) shows the radial distance from the home for four sample trajectories. In Fig. 10(b) the time-dependent autocorrelation function of distances corresponding to the trajectories in Fig. 10(a) is plotted. In the simulations, we calculated

$$
C_{r r}(\Delta t)=\lim _{T \rightarrow \infty} \frac{1}{T} \int_{0}^{T}[r(t)-\langle r\rangle][r(t+\Delta t)-\langle r\rangle] d t .
$$

Further on we assumed stationarity and have set $\langle r\rangle=2 v_{0} / \kappa$ in agreement with (27). We underline the good qualitative agreement of the presented results from simulations with the findings for the fruit fly $[5,12]$. To compare Fig. 10(b) with Fig. 6.B of [5] we consider $v_{0}=10 \mathrm{~mm} / \mathrm{s}$ as given by [5] as a realistic value. With this value, the lag in millimeters provided in Fig. 6.B of [5] becomes a timescale if divided by the velocity.

Figure 11 shows the sojourn time distribution to a home of the size $r_{s}=0.1$. The particles start at the home with $r(t=0)=r_{s}$ and the initial angles $z_{0}$ are uniformly distributed between $z_{0} \in[0, \pi / 2]$ and $z_{0} \in[-\pi,-\pi / 2]$. Those initial conditions correspond to being at the home and moving away from the home in the steady state. Note that equidistribution of $z_{0}$ does not imply a uniform distribution of the $X$ values. The particle is considered to have returned to the home if it touches the extended home for the first time, i.e., $r\left(t>t_{0}\right)=r_{s}$.

The parameters are chosen to be consistent with the previous examples. Figure 11 shows a low noise intensity case $\sigma^{\alpha}=$ 0.01 , while the inset on the right displays a rather high noise intensity $\sigma^{\alpha}=0.5$. The left inset corresponds to the deterministic case. The speed is $v_{0}=1$ and the coupling to the home $\kappa=1$ is constant. The distribution of sojourn times for the deterministic case can be seen in the left inset. Note that particles start returning to the home after $t_{p} \approx 9.5$. The

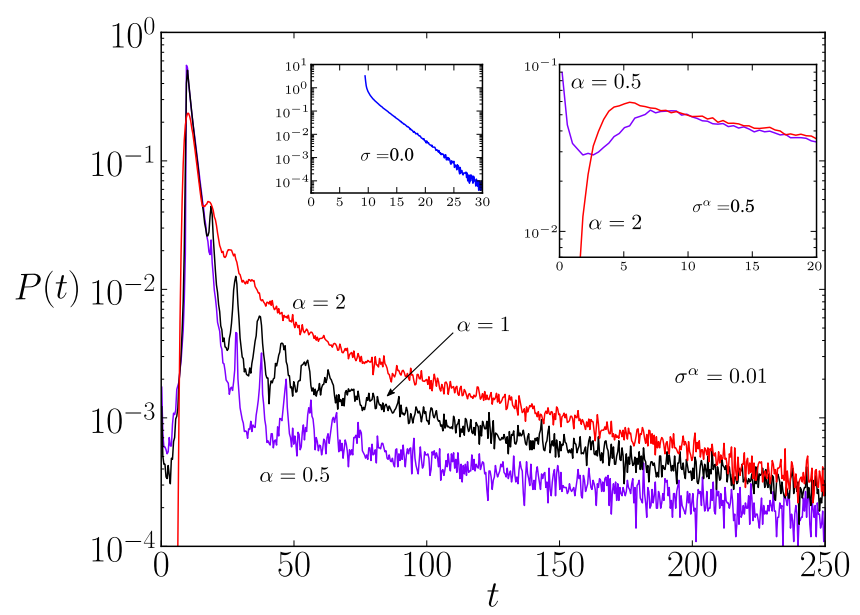

FIG. 11. Return time to the home for three different noise types and three noise strengths. The home is assumed to be extended. Particles start at a distance $r(t=0)=0.1$ from the home and are considered to have returned when the distance is $r=0.1$ again. See the text for a description of the insets. The other parameters are $v_{0}=1$ and $\kappa=1$. 

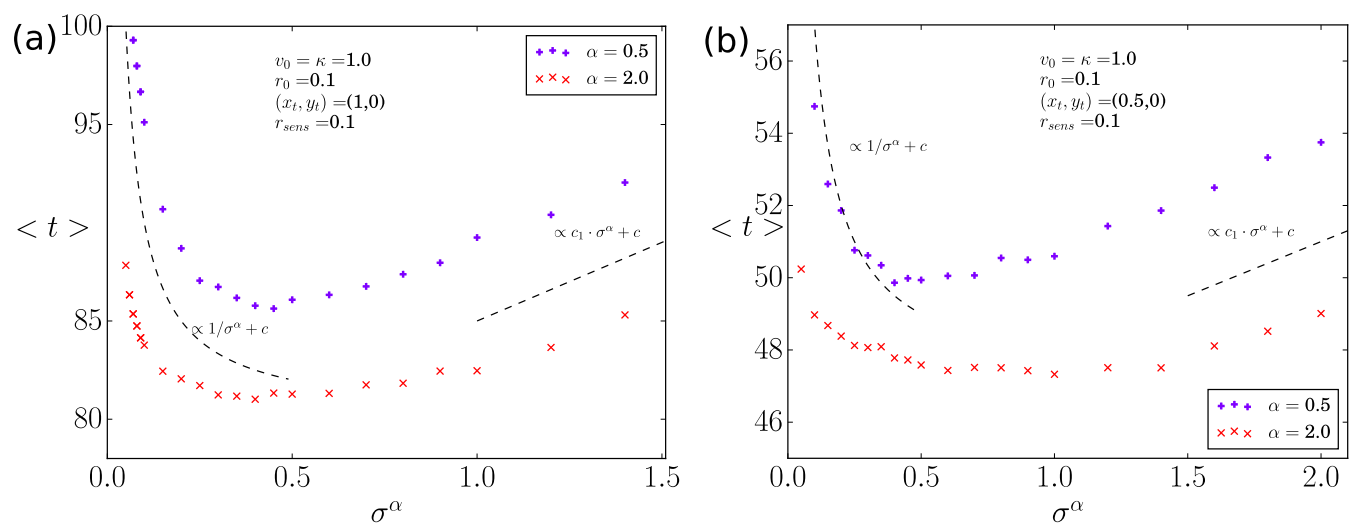

FIG. 12. Mean time $\langle t\rangle$ until a new spot $\left(x_{t}, y_{t}\right)$ at a given position in the $(x, y)$ plane is found dependent on the noise intensity with (a) $\left(x_{t}=1, y_{t}=0\right)$ and (b) $\left(x_{t}=0.5, y_{t}=0\right)$.

particles which return first correspond to the initial condition $r_{0}=r_{s}$ and $z_{0}=\pi / 2$. All others initially take a longer period of time. For $t>t_{p}$ the distribution sharply descends and takes an exponential form.

In Fig. 11 sojourn time distributions are plotted for three noise types. All three noise types display a sharp peak at approximately $t_{p} \approx 9.5$. This time $t_{p}$ is the time a deterministic particle with $r_{0}=r_{s}$ and $z_{0}=\pi / 2$ needs to return to the home $r(t)=r_{s}$. The shape of the peak is influenced by the noise type: For the Gaussian noise $\alpha=2$ the peak is rather smooth compared to the Cauchy noise $\alpha=1$ and even more so for $\alpha=0.5$. For the lowest displayed noise the decay of the first peak approximately resembles the exponential decay of the deterministic particles.

The behavior at the first peak can be understood by considering Fig. 8. Figures 8(a) and 8(b), corresponding to $\alpha=0.5$ and $\alpha=2.0$, display the sample trajectories in the $(r, X)$ plane. Here the noise types act very distinct on the motion of the particles: While a Gaussian noise almost at every instant in time causes a comparatively small change in the deterministic trajectory $X$, the other extreme, the $\alpha=0.5$ case, rarely cause a change, but if it causes a change, the change can be large. This behavior causes in Fig. 11 an almost deterministic shape of the first peak for $\alpha=0.5$ and the smearing out of the peak for Gaussian white noise. Before the peak the different noise distributions also cause distinct behavior. For Gaussian noise basically no particle returns for times roughly $t<5$, while in the other cases some particles immediately return. As Lévy noise with smaller $\alpha$ increases the probability of large sudden changes, this different behavior can be understood. For non-Gaussian noise the direction of motion of the particles can suddenly jump by $\pi$ and therefore allowing an immediate return of the particle.

After the first peak several other peaks follow in all cases, but with different intensity. In fact, already in the first peak a second peak is visible. Those peaks correspond to multipliers of $n t_{p} n \in N$. Best visible are those peaks for $\alpha=0.5$ up to roughly the correlation time $\tau$.

A high noise intensity causes almost the same shape of the time distribution for all noise types. Differences only remain at small times $t \ll t_{p}$, as can be seen in the inset on the right. Both curves seem to decay in the same way and therefore only a small time interval is shown.

\section{B. Mean first hitting time}

For insects an oscillatory motion around a given home is often considered to be foraging; the question arises how fast a particle in our model can discover a food source. We assume our searcher has a sensing radius $r_{\text {sens. }}$. This sensing radius is small compared to the length scale $r_{c}$ of our system; we choose $r_{\text {sens }}=0.1 \ll r_{c}=1$. We expect the sensing radius to be small compared to $r_{c}$ as otherwise not much new space can be discovered. We perform simulations of particles starting close to the home $r(t=0)=r_{0}=r_{\text {sens }}$, with uniformly distributed heading directions and position angles, and determine the mean first hitting time $\langle t\rangle$ until the food source is discovered. The localized target or the new food source is placed at $\left(x_{t}, y_{t}\right)=$ $(1,0)$ and at $\left(x_{t}, y_{t}\right)=(0.5,0)$. Localized means that the target is pointlike. We choose the first spot at a distance of $r_{c}$, as every deterministic bounded trajectory crosses this distance and the spatial distribution (27) possesses maximal probability there. As the second spot we choose a closer one to investigate how the behavior of the mean first hitting time changes. The searcher usually returns several times to the home until the food source is discovered. Figure 12 shows the mean first hitting time $\langle t\rangle$ for a given new food spot $\left(x_{t}, y_{t}\right)$ dependent on the inverse relaxation time $1 / \tau$. Since we have set $v_{0}=1$, the latter is, according to Eq. (33), proportional to the scaled noise intensity $\sigma^{\alpha}$.

In both pictures an optimal noise strength can be seen, at which the mean first hitting time $\langle t\rangle$ is minimal. This optimal time depends on the relaxation time $\tau$ but also on the distance between the food source and the home. The optimal noise strength depends on the distance where the target is situated and decays with growing distance.

The nonmonotonic dependence of the hitting times can be explained as resulting from two counteracting effects originated by the noise. Considering a deterministic motion $\sigma=0$, not every searcher will hit the target as most of the radial unbounded trajectories will miss it. For bounded trajectories the mean time will be proportional to the period length. We recall here that the sensing radius is small, so even after the accumulated apsidal precession is larger than $2 \pi$ the particle is likely not to have found the food source. In the deterministic case the mean first hitting time diverges. With low noise present the searcher can switch deterministic trajectories. Now particles following a deterministic trajectory 


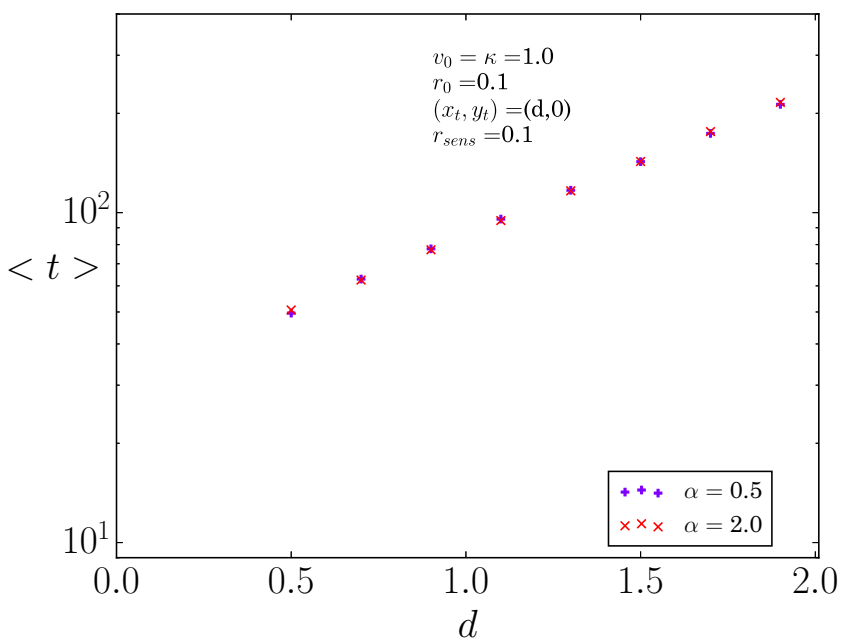

FIG. 13. Mean time $\langle t\rangle$ for a fixed noise intensity $\sigma^{\alpha}=0.4$ dependent on the spot distance $d$, with $\left(x_{t}=d, y_{t}=0\right)$.

that never hits the target or that takes extremely long to return might arrive earlier through switching. This redistribution is proportional to the timescale of the integral of motion $X$ given by (32). Correspondingly, the mean first hitting time decays proportionally to $\tau$ from (33). The mean first hitting time is always larger than the relaxation time $\tau$.

The second counteracting process starts to act at a higher noise. Now the deterministic motion can be practically neglected and slow diffusive motion of the searcher dominates. The timescale for diffusive motion is given by (B5) and (35). Therefore, the time after which a certain distance $\Delta r$ has been reached on average by this diffusion can be estimated as

$$
t_{\text {diff }}=\frac{\left\langle\Delta r^{2}\right\rangle}{4 D_{\text {eff }}}=\frac{\sigma^{\alpha}}{2 v_{0}^{2+\alpha}}\left\langle\Delta r^{2}\right\rangle .
$$

Thus, for high noise intensity the hitting time starts growing proportionally to $\sigma^{\alpha}$. Since the latter scales proportionally to $1 / \tau$, we consolidated the second noise-induced effect governing the increasing hitting times.

In our numerical simulations Gaussian white noise always performed slightly better than all other noise types $(\alpha<2)$. When changing the noise parameter $\alpha$ to lower values we observed only a small increase of the mean time $\langle t\rangle$. So different turning statistics seem not to significantly improve the local search in our model, if $\langle t\rangle \geqslant \tau$.

This result is contrary to the result for freely moving Daphnia [23] during a global search, where an optimal turning angle distribution for search was found. However, for a searcher with an uncertainty of the exact position angle, the turning angle distribution can significantly influence the success of returning home [28].

Figure 13 shows the mean first hitting time for fixed noise strength $\sigma^{\alpha}=0.4$ dependent on the spot distance $d$. As can be seen, the times grow exponentially fast with the distance. We point out that for each of the considered distances we observed a nonmonotonic dependence of the mean hitting times on the noise intensity $\sigma^{\alpha}$.

We mentioned in Sec. IV B that we consider $v_{0}=10 \mathrm{~mm} / \mathrm{s}$ and $\kappa=0.5 \mathrm{~s}^{-1}$ and therefore $r_{c}=20 \mathrm{~mm}$ as realistic values for a fly. We mention here that for such values and a food source at a spot distance $d=20 \mathrm{~mm}$ the optimal time becomes $\langle t\rangle=160 \mathrm{~s}$ and with $d=40 \mathrm{~mm}$ the optimal time becomes $\langle t\rangle=800 \mathrm{~s}$.

\section{COUPLING DEPENDENT ON DISTANCE}

In this section we generalize the model through replacing the coupling parameter $\kappa$ to the home by a distancedependent coupling $\kappa(r)$, with $r=\sqrt{x^{2}+y^{2}}$, when considering Cartesian coordinates. By doing so, we cover a broader class of spatial distributions and deterministic trajectories and the model might describe also more complex motions with preferred paths. It is straightforward to adjust the so far determined properties: the deterministic trajectories (13), the spatial distribution (27), and the relaxation time (33).

We introduce the time evolution of the direction of the velocity $\vec{v}$

$$
\dot{\theta}=\kappa(r) \sin (\theta-\beta)+\frac{\sigma}{v_{0}} \xi(t),
$$

with coupling $\kappa(r)$. We require that the function $\kappa(r)$ has no singularity for all distances including $r=0$. This way we ensure that at the origin $(x, y)=(0,0)$ the system in Cartesian coordinates is well defined. In addition to ensuring the existence of a steady state, the spatial density of searchers will be normalizable. This will set another condition on $\kappa(r)$.

The time evolution of the angle $z$ between the direction of motion $\theta$ and the position of the home $\beta$ is now given by

$$
\dot{z}=-\left(\frac{v_{0}}{r}-\kappa(r)\right) \sin (z)
$$

It follows for the deterministic trajectories that

$$
\sin [z(r)] \exp \left(-\frac{U(r)}{v_{0}}\right) r=X=\sin \left(z_{0}\right) \exp \left(-\frac{U\left(r_{0}\right)}{v_{0}}\right) r_{0}
$$

holds, wherein we have defined

$$
U(r)=\int^{r} d r^{\prime} \kappa\left(r^{\prime}\right) .
$$

Again, a particle with an initial angle $z_{0}=0$ or $z_{0}=\pi$ will move on a straight line. There also exist a minimal and a maximal distance from the home, with $\sin (z)= \pm 1$. Previously, in Eq. (13) we could set $r_{0}$ such that the integral of motion $X\left(z_{0}\right)$ only depended on the initial angle $z_{0}$. The value of $X\left(z_{0}\right)$ represented a trajectory in the $(r, z)$ plane, or in the $(r, X)$ plane in a unique way. Now this might no longer be true. Now the variable $X\left(r_{0}, z_{0}\right)$ might be dependent on the initial position and the initial angle, if one wants to uniquely identify a trajectory in the $(r, z)$ plane and have $z_{0}$ as a parameter. One could, however, set the initial angle $z_{0}= \pm \pi / 2$ and then the variable $X\left(r_{0}\right)$ would still be dependent on only one parameter and be unique for the trajectories in the $(r, z)$ plane, but we choose the initial angle $z_{0}$ to be a parameter. Figure 14 displays such a case. Figure 14(a) shows the steady-state PDF. It is obtained from (24) by replacing $\kappa$ with $\kappa(r)$ and following the same steps as in Sec. IV B,

$$
P_{0}(r, z)=c r \exp \left(-\frac{U(r)}{v_{0}}\right),
$$



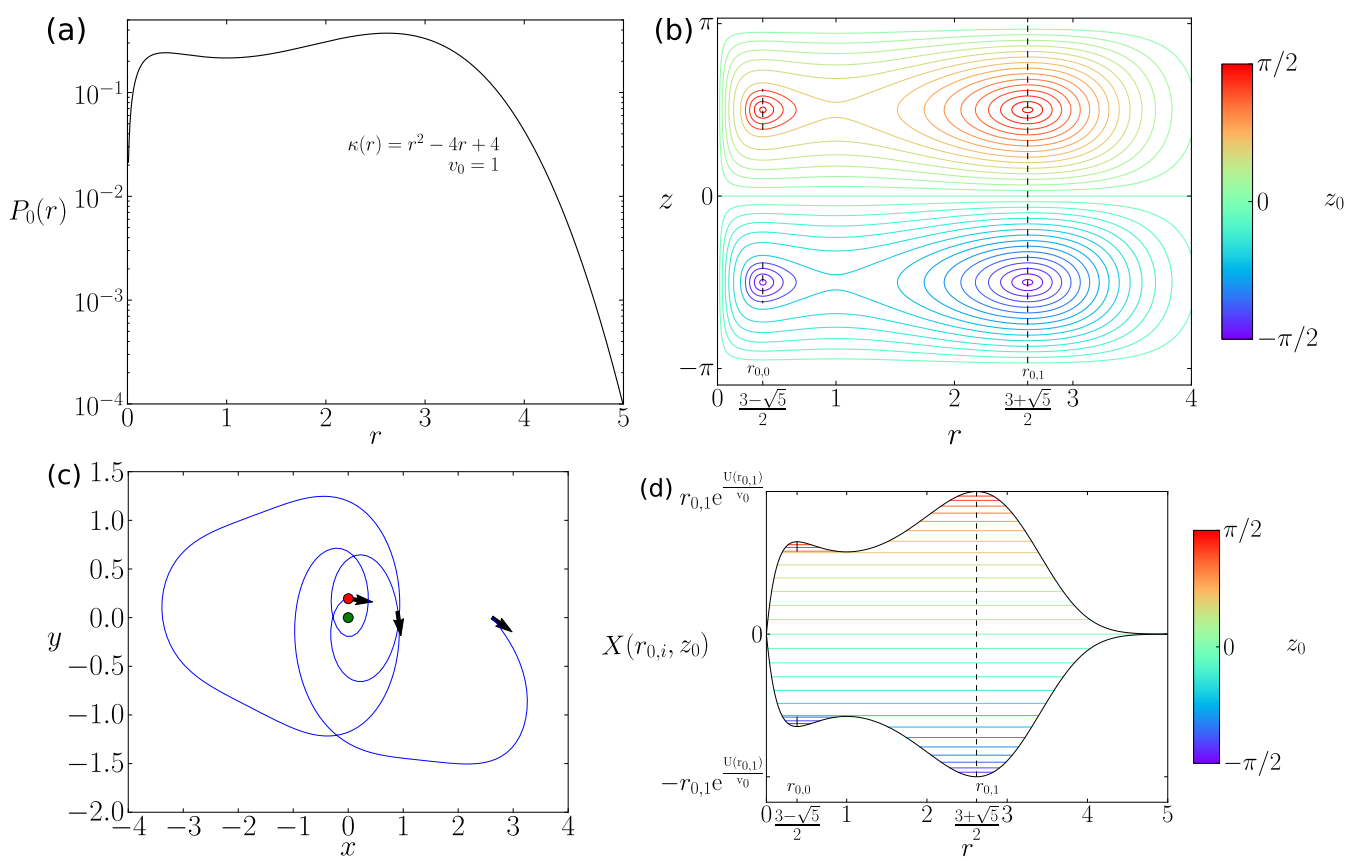

FIG. 14. Example of distance-dependent coupling strength $\kappa(r)$. (a) Steady-state spatial distribution $P_{0}$. (b) Corresponding deterministic trajectories in the $(r, z)$ plane, with initial condition $r_{0}$, as indicated by dashed lines and initial angle $z_{0}$ according to the color bar. (c) Sample trajectory in the $(x, y)$ plane without noise corresponding to the blue separatrix in the $(r, z)$ plane. (d) Trajectories in the $(r, X)$ plane with the initial condition $r_{0}$, as indicated by dashed lines and initial angle $z_{0}$ according to the color bar.

which again is independent of the noise. It reads in Cartesian coordinates

$$
P_{0}(x, y)=c \exp \left(-\frac{U\left(\sqrt{x^{2}+y^{2}}\right)}{v_{0}}\right)
$$

with $c$ the normalization constant. This sets the second condition on $\kappa(r)$ as we require the normalization to be possible.

The noise still acts perpendicularly on the deterministic trajectory $X$ causing a switching and the ensemble average $\langle X\rangle$ still follows the exponential decay from Eq. (32), with the same relaxation time as given before (33). The overdamped dynamics for the radial transition $\operatorname{PDF} P\left(r, t \mid r_{0}, t_{0}\right)$,

$$
\frac{\partial}{\partial t} P=D_{\text {eff }} \frac{\partial}{\partial r}\left[\frac{\partial}{\partial r} P-\left(\frac{1}{r}-\frac{\kappa(r)}{v_{0}}\right) P\right],
$$

has (43) as an asymptotic steady PDF. As above, the radial relaxation again slows down with increased noise strength. Likewise in the situation with constant $\kappa$, only $\tau$ or $D_{\text {eff }}$ expresses the influence of the various $\alpha$ values of the noise.

Figure 14 gives an example of a space-dependent coupling $\kappa(r)$. We choose $\kappa(r)=r^{2}-4 r+4$ such that the steady-state PDF exhibits two maxima. This can be seen in Fig. 14(a). In Fig. 14(b), the deterministic trajectories are shown in the $(r, z)$ plane, with two spatial initial conditions $r_{0, i}(i=0,1)$ as indicated by the two dashed lines and the initial angle $z_{0}$ according to the color bar. Due to the quadratic term in the coupling, trajectories are significantly shorter than in the initial model since the coupling to the home-pointing direction increases with the distance from the home.
Extremal points of radial probability follow from $d P_{0} / d r=$ 0 for the considered coupling from

$$
1-\frac{r}{v_{0}} \kappa(r)=0 .
$$

Solutions are easily found. The minimal probability is found around $r_{1}=1$ and the maximal one at the two distances $r_{2,3}=$ $(3 \pm \sqrt{5}) / 2$, as shown in Fig. 14(a). In the $(r, z)$ plane [see Fig. 14(b)], these distances are connected with the fixed points of the deterministic flow of trajectories located at angles $z=$ $\pm \pi / 2$. The fixed points at $r_{1}$ are of saddle type, whereas those at the two other distances are centers. They always correspond to a circular solution for the deterministic trajectories in the $(x, y)$ plane since at those points the angular and radial velocities $\dot{z}=0$ and $\dot{r}=0$, respectively, vanish.

Small changes in the initial angle $z_{0}$ around the circular solutions in the $(x, y)$ plane at the maxima $r_{2,3}$ correspond to trajectories in the $(x, y)$ plane similar to Fig. 4(a). For initial angles $z_{0}$ close to 0 or $\pm \pi$, trajectories in the $(x, y)$ plane will be comparable to Fig. 4(b), but shorter with faster turnings. Between the two mentioned solutions, a separatrix lies. Figure $4(c)$ is a trajectory in the $(x, y)$ plane close to the separatrix. This solution exists if the initial angle $z_{0}= \pm \pi / 2$ at the minimum is slightly changed. The resulting trajectory in the $(x, y)$ plane rotates two times around the home before reaching the maximal distance twice. The noise facilitates again switchings between trajectories.

If the steady-state PDF of the position can be experimentally measured it can be fitted to our solution (44), thus determining the coupling strength $\kappa(r)$ to the home. Having found a suitable dependence, the relaxation time $\tau$ can be determined by the ensemble or time average of the variable $X$ through measuring $r(t)$ and $z(t)$. Fitting experimental data thus allows one to 
determine the relaxation time $\tau$ and therefore the noise strength $\sigma^{\alpha}$ of the model.

\section{CONCLUSION}

We laid the foundation for a minimal stochastic model for a local searcher which was motivated by experimental observations of the stochastic oscillatory motion of insects around a given home. The main ingredients of this minimal model are the constant speed of the searcher and stochastic angular variation, which only requires the knowledge of the position angle and the heading direction, which allows the particle to explore the vicinity of the given home. The specific interaction with this home results in an exploration of the neighborhood around the home and an attraction towards it dependent on the mutual orientations of the position and heading vectors.

The model was formulated with four parameters: $\kappa$ defines the strength of interaction with the home, $v_{0}$ is the speed of the searcher, and $\sigma$ stands for the intensity of the noise. Since the observed turning angle behavior in experiments such as fruit flies can be of non-Gaussian statistics, we introduced $\alpha$-stable noise as the source of randomness. The corresponding parameter $0<\alpha \leqslant 2$ as the fourth parameter allows one to vary the support of the noise source between special types of noise as Gaussian, Lorentzian, etc.

The introduced model showed qualitative agreement with the behavior of insects. The advantage of our model is the analytical and simple numerical tractability. In consequence, we were able to discuss typical behavior of the trajectories and of characteristic times. For example, we found the characteristic return times in the noise-free case and obtained an apsidal precession of the oscillatory trajectories reminiscent of celestial motion. The analysis of the models allowed us to discuss in detail the deterministic properties and the effects originating through the addition of different symmetric whitenoise sources.

The inclusion of noise has a stabilizing effect on the system since unstable trajectories disappear. Generally, trajectories start to randomize. This is manifested by the noise-dependent relaxation time $\tau$ that is proportional to $1 / \sigma^{\alpha}$. For larger times the stochastic dynamics has forgotten its initial directions and trajectories have spread over all possible orbits. This investigation has concentrated on the relaxation of the deterministic integral of motion $X$. Its first moment conditioned on initial values has decayed at times larger $\tau$.

At high noise the particles start to perform diffusive motion. Like every active stochastic particle, the corresponding effective diffusion coefficient depends inversely proportionally on $\sigma$. We derived for the nonlinear model the overdamped Smoluchowski equation outgoing from the FPE for all $\alpha$ values. It describes the stochastic dynamics on timescales much larger than the noise-dependent time and $\tau$. Except for $\tau$, the Smoluchowski equation is independent of the noise characteristics.

We obtained analytically the steady-state spatial distribution $P_{0}$ which appeared to be identical for all different kinds of $\alpha$-stable noise [see Eq. (27)]. In particular, it depends neither on $\sigma$ nor on $\alpha$. Distances are exponentially distributed and the width is determined by the ratio $v_{0} / \kappa$, which is just a characteristic length for the quasiperiodic excursions which the stochastic trajectories perform.

We found in our model an optimal noise strength for finding a new spot in the minimal average time $\langle t\rangle$. This optimal average time is distance dependent. The searcher finds on average the new spot always faster with noise in the angular dynamics. This is the result of the relaxation towards a probabilistic population of all possible trajectories which determines the greater success of the stochastic searcher. For lower noise this process is governed by the noisy periodic motion and after the relaxation time the stationary PDF is established. However, for higher noise the relaxation is proceeded by diffusive search. If an approximate distance of a spot to discover is given, we expect a good choice that this length equals the length scale $r_{c}$ of our system. We found in our model only an insignificant dependence on the turning angle distribution, expressed through the noise type. This result is in contrast to results for a global search [23]. Other work shows, however, that the probability to return to the home strongly depends on the noise type if an uncertainty of the position angle exists [28].

Another advantage of our model is that we could generalize the model to distance-dependent coupling $\kappa(r)$, thus allowing us to express a large class of radial symmetric spatial steadystate distributions [see Eq. (43)] and corresponding spatial trajectories. All models of this class exhibit the timescale $\tau$, so we expect the existence of an optimal noise strength for the general model. The long-time behavior of systems with distance-dependent coupling follows again a Smoluchowski equation uniformly for all $\alpha$ 's.

We underline that our findings are applicable to a broad class of stochastic searching units such as insects and autonomous vehicles. Here we considered individual searchers. The investigation concentrated on the interaction with the given home and on temporal scales to find new food sources. Further research on interacting searchers and their cooperative behavior is needed.

\section{ACKNOWLEDGMENTS}

This work was supported by the Deutsche Forschungsgemeinschaft via Grant No. IRTG 1740 and by the São Paulo Research Foundation via Grant No. 2015/50122-0 and No. 2017/04552-9. L.S.-G. thanks Alexander Neiman and Ohio University in Athens, Ohio for hospitality and support. The authors thank Fabian Baumann for fruitful discussions.

\section{APPENDIX A: DERIVATION OF THE STOCHASTIC $X$ DYNAMICS}

Here we derive the linear differential equation for the averaged value of $X$ as defined by

$$
\int_{0}^{\infty} d r \int_{-\pi}^{\pi} d z X(r, z) P\left(r, z, t \mid r_{0}, z_{0}, t_{0}\right)
$$

[compare Eq. (30)]. This characterizes the relaxation of the stochastic $X(r, z, t)$ dynamics. It is dissipative in contrast to 
the deterministic behavior. To obtain the dynamics for the average conditioned to the initial values $r_{0}, z_{0}$ we multiply the FPE (24) for the transition PDF $P=P\left(r, z, t \mid r_{0}, z_{0}, t_{0}\right)$ by $X$ from (13) and integrate over $r$ and $z$ :

$$
\int_{0}^{\infty} d r \int_{-\pi}^{\pi} d z X(r, z) \frac{\partial}{\partial t} P=\int_{0}^{\infty} d r \int_{-\pi}^{\pi} d z X(r, z)\left[-v_{0} \frac{\partial}{\partial r} \cos (z)+\frac{\partial}{\partial z}\left(\frac{v_{0}}{r}-\kappa\right) \sin (z)+\left(\frac{\sigma}{v_{0}}\right)^{\alpha} \frac{\partial^{\alpha}}{\partial|z|^{\alpha}}\right] P .
$$

The first term on the rhs after partial integration over $r$ together with the second term partially integrated over $z$ results in an expression which vanishes if $X(r, z)$ is inserted,

$$
\int_{0}^{\infty} d r \int_{-\pi}^{\pi} d z\left[v_{0} \cos (z) P \frac{\partial}{\partial r} X-\left(\frac{v_{0}}{r}-\kappa\right) \sin (z) P \frac{\partial}{\partial z} X\right]=0 .
$$

Thus we are left with

$$
\frac{d}{d t}\langle X\rangle=\left(\frac{\sigma}{v_{0}}\right)^{\alpha} \int_{0}^{\infty} d r \int_{-\pi}^{\pi} d z X \frac{\partial^{\alpha}}{\partial|z|^{\alpha}} P\left(r, z, t \mid r_{0}, z_{0}, t_{0}\right)
$$

We express the PDF through its Fourier transform

$$
P\left(r, z, t \mid r_{0}, z_{0}, t_{0}\right)=(2 \pi)^{-1} \int_{-\infty}^{\infty} d k \exp (-i k z) P\left(r, k, t \mid r_{0}, z_{0}, t_{0}\right) .
$$

Its introduction in (A4) and taking the angle $z$ to be unwrapped yields

$$
\frac{d}{d t}\langle X\rangle=-\frac{1}{2 \pi}\left(\frac{\sigma}{v_{0}}\right)^{\alpha} \int_{0}^{\infty} d r \int_{-\infty}^{\infty} d z \int_{-\infty}^{\infty} d k \exp (-i k z) X(r, z)|k|^{\alpha} P\left(r, k, t \mid r_{0}, z_{0}, t_{0}\right) .
$$

Including the definition of $X$ and performing the $z$ integration leads to

$$
\frac{d}{d t}\langle X\rangle=-\left(\frac{\sigma}{v_{0}}\right)^{\alpha} \frac{1}{2 \pi} \int_{0}^{\infty} d r \int_{-\infty}^{\infty} d k \frac{1}{2 i}[\delta(k-1)-\delta(k+1)] r \exp \left(-\frac{r}{r_{c}}\right)|k|^{\alpha} P\left(r, k, t \mid r_{0}, z_{0}, t_{0}\right),
$$

with the $\delta$ functions originated by the sine function in $X$. Eventually, we have a look at the definition of the average (A1). Therein we also perform the Fourier transform and take an unwrapped angle $z$ which gives

$$
\langle X\rangle=\frac{1}{2 \pi} \int_{0}^{\infty} d r \int_{-\infty}^{\infty} d k \frac{1}{2 i}[\delta(k-1)-\delta(k+1)] r \exp \left(-\frac{r}{r_{c}}\right) P\left(r, k, t \mid r_{0}, z_{0}, t_{0}\right) .
$$

Comparing Eqs. (A5) and (A6) shows that after performing the $k$ integration both equations are identical up to the factor in front of (A5); therefore, it follows that

$$
\frac{d}{d t}\langle X\rangle=-\frac{1}{\tau}\langle X\rangle
$$

This equation is solved by (32) with $\langle X\rangle\left(t_{0}\right)=X_{0}=X\left(r_{0}, z_{0}\right)$ the initial value of $\langle X\rangle$.

One finds the same relaxation time also by using the time-dependent eigenfunction of the FPE (24). It is solved by

$$
e_{1}(r, z, t) \propto \sin (z) r^{2} \exp \left(-2 \frac{\kappa}{v_{0}} r-\frac{t}{\tau}\right),
$$

with the eigenvalue $\lambda_{1}=1 / \tau$. It describes the relaxation of the integral of motion $X$ in the case in which $\alpha$-stable noise is present in the angular dynamics.

\section{APPENDIX B: DERIVATION OF THE OVERDAMPED SMOLUCHOWSKI EQUATION}

Following [29,30], which discussed an overdamped description of freely diffusing active particles with Gaussian white noise, we define the Fourier components

$$
P_{n}(r, t)=\int_{-\pi}^{\pi} d z \exp (i n z) P(r, z, t), \quad n=0, \pm 1, \pm 2, \ldots
$$

For simplicity, we omit the initial states in the transition PDF $P$ and in the components. Note that the zeroth component with $n=0$ equals the marginal spatial PDF $P(r, t)$, for which we want to derive an approximative equation as well as get the necessary conditions for its validity.

We multiply the FPE (24) from the left with $\exp (i n z)$ and integrate over $z$. We obtain a set of coupled partial differential equations for the Fourier amplitudes

$$
\frac{\partial}{\partial t} P_{n}=-\frac{v_{0}}{2} \frac{\partial}{\partial r}\left(P_{n+1}+P_{n-1}\right)-\frac{n}{2}\left(\frac{v_{0}}{r}-\kappa\right)\left(P_{n+1}-P_{n-1}\right)+\left(\frac{\sigma}{v_{0}}\right)^{\alpha}|n|^{\alpha} P_{n} .
$$


Afterward, we eliminate the last term on the rhs by substituting $P_{n}=\exp \left(-|n|^{\alpha} t / \tau\right) P_{n}^{\prime}$, with $\tau$ from (33):

$$
\begin{aligned}
\frac{\partial}{\partial t} P_{n}^{\prime}= & -\frac{v_{0}}{2} \frac{\partial}{\partial r}\left[\exp \left(-\left(|n+1|^{\alpha}-|n|^{\alpha}\right) \frac{t}{\tau}\right) P_{n+1}^{\prime}+\exp \left(-\left(|n-1|^{\alpha}-|n|^{\alpha}\right) \frac{t}{\tau}\right) P_{n-1}^{\prime}\right] \\
& -\frac{n}{2}\left(\frac{v_{0}}{r}-\kappa\right)\left[\exp \left(-\left(|n+1|^{\alpha}-|n|^{\alpha}\right) \frac{t}{\tau}\right) P_{n+1}^{\prime}-\exp \left(-\left(|n-1|^{\alpha}-|n|^{\alpha}\right) \frac{t}{\tau}\right) P_{n-1}^{\prime}\right] .
\end{aligned}
$$

Considering the index $n=0$, we note that for $n=0$ the components $P_{0}^{\prime}$ and $P_{0}$ converge and equate to the marginal distance PDF $P(r, t)$.

We take another partial time derivative of $P_{0}^{\prime}$ in (B3). In the obtained relation we replace $P_{1}^{\prime}(t)$ using (B3) with $n=0$ on the left-hand side. This leads to an expression containing first and second derivatives of $P_{0}^{\prime}$ and terms with $P_{ \pm 2}^{\prime}$ :

$$
\left.\frac{\partial}{\partial t^{2}} P_{0}^{\prime}+\frac{1}{\tau} \frac{\partial}{\partial t} P_{0}^{\prime}=\frac{v_{0}^{2}}{2} \frac{\partial}{\partial r}\left[\frac{\partial}{\partial r} P_{0}^{\prime}-\left(\frac{1}{r}-\frac{\kappa}{v_{0}}\right) P_{0}^{\prime}\right]+\frac{v_{0}^{2}}{4} \exp \left(-|2|^{\alpha} \frac{t}{\tau}\right) \frac{\partial}{\partial r}\left[\frac{\partial}{\partial r}\left(P_{2}^{\prime}+P_{-2}^{\prime}\right)\right)+\left(\frac{1}{r}-\frac{\kappa}{v_{0}}\right)\left(P_{2}^{\prime}-P_{-2}^{\prime}\right)\right] \text {. }
$$

For $t \gg \tau$ the terms containing $P_{ \pm 2}$ vanish and as was discussed in [29,30] the second time derivative containing a ballistic part of the motion can be also neglected if the limit of $v_{0}^{2} \tau$ for small $\tau$ remains finite. Therefore, we are left, under these conditions, with the overdamped description

$$
\frac{\partial}{\partial t} P(r, t)=D_{\mathrm{eff}} \frac{\partial}{\partial r}\left[\frac{\partial}{\partial r} P(r, t)-\left(\frac{1}{r}-\frac{\kappa}{v_{0}}\right) P(r, t)\right]
$$

with the effective diffusion coefficient

$$
D_{\text {eff }}=\frac{v_{0}^{2} \tau}{2}
$$

The resulting Smoluchowski equation determines the overdamped dynamics of the spatial transition PDF $P\left(r, t \mid r_{0}, t_{0}\right)$ as used in Sec. IV C. Since its validity is bounded to timescales $t \gg \tau$, the initial angle $z_{0}$ is forgotten and the PDF depends on $z_{0}$, further on. Small $\tau$ implies high noise or small velocities in agreement with (33).

\section{APPENDIX C: MECHANICS OF THE SEARCHER}

Here we elaborate some similarities of the deterministic dynamics to the celestial mechanics. The motion of the particle is reminiscent of the planetary motion around a central body in an attracting potential. However, we first underline the main differences. In our problem we always deal with constant speed of the particle, which is another integral of motion in the problem.

In consequence, the kinetic energy of the particle becomes [compare Eq. (16)]

$$
E_{\text {kin }}=\frac{1}{2}\left(\frac{d r}{d t}\right)^{2}+v_{0}^{2} \sin ^{2}(z)=\frac{1}{2} v_{0}^{2} .
$$

After replacement of the $\sin (z)$ item by the constant integral of motion $X(r, z)$, the $z(t)$ variable disappears and the energy reads

$$
E_{\mathrm{kin}}=\frac{1}{2}\left(\frac{d r}{d t}\right)^{2}+\frac{1}{2} v_{0}^{2}\left(\frac{X}{r}\right)^{2} \exp \left(2 \frac{r}{r_{c}}\right)=\frac{1}{2} v_{0}^{2} .
$$

This equation could be reinterpreted as describing the full mechanical energy for a particle moving in a central force field at distance $r(t)$ and with effective potential

$$
U_{\text {eff }}(r)=\frac{1}{2} v_{0}^{2}\left(\frac{X}{r}\right)^{2} \exp \left(2 \frac{r}{r_{c}}\right)
$$

The effective central force acting on the particle is

$$
F_{\text {eff }}(r)=-\frac{\partial}{\partial r} U_{\text {eff }}(r)=\left(\frac{X}{r}\right)^{2}\left(\frac{1}{r}-\frac{1}{r_{c}}\right) \exp \left(2 \frac{r}{r_{c}}\right) \text {. }
$$

So the motion of the particle is conservative and it moves through an effective central field, with the potential energy given by (C3). This effective potential becomes infinity at $r=$ 0 and if $r \rightarrow \infty$. Hence, the particle performs only bounded oscillatory motion with full energy $v_{0}^{2} / 2$. At the perihelion and aphelion with $r_{\max / \min }$, respectively, the potential energy is extremal $U_{\text {eff }}=1 / 2$, as the radial velocity vanishes at those distances. The force changes between attraction and repulsion at $r=r_{c}$, where the radial velocity of the particle is maximal.
[1] R. Klages, in Diffusive Spreading in Nature, Technology and Society, edited by A. Bunde, J. Caro, J. Kärger, and G. Vogl (Springer, Cham, 2017), pp. 49-69.

[2] M. Mittelstaedt and H. Mittelstaedt, Naturwissenschaften 67, 566 (1980).

[3] K. Cheng, Psychology of Learning and Motivation, Psychology of Learning and Motivation Vol. 33 (Academic, New York, 1995), pp. 1-21.
[4] R. F. Wang, Cognitive Vision, Psychology of Learning and Motivation Vol. 42 (Academic, New York, 2003), pp. 109-156.

[5] I. S. Kim and M. H. Dickinson, Curr. Biol. 27, 2227 (2017).

[6] J. Zeil, Cur. Opin. Neurobiol. 22, 285 (2012).

[7] J. D. Seelig and V. Jayaraman, Nature (London) 521, 186 (2015).

[8] J. Green, A. Adachi, K. K. Shah, J. D. Hirokawa, P. S. Magani, and G. Maimon, Nature (London) 546, 101 (2017). 
[9] R. J. Vickerstaff and T. Merkle, J. Theor. Biol. 307, 1 (2012); see also R. J. Vickerstaff, Evolving dynamical system models of path integration, Ph.D. thesis, University of Sussex, 2007.

[10] R. Wehner and M. V. Srinivasan, J. Comp. Physiol. A 142, 315 (1981).

[11] R. Wehner, B. Michel, and P. Antonsen, J. Exp. Biol. 199, 129 (1996).

[12] B. el Jundi, Curr. Biol. 27, R748 (2017).

[13] S. Chien and K. L. Wagstaff, Sci. Robot. 2, eaan4831 (2017).

[14] J. V. Hook, P. Tokekar, E. Branson, P. G. Bajer, P. W. Sorensen, and V. Isler, in Experimental Robotics: The 13th International Symposium on Experimental Robotics, edited by J. P. Desai, G. Dudek, O. Khatib, and V. Kumar (Springer, Heidelberg, 2013), pp. 859-873.

[15] Y. Girdhar, A. Xu, B. B. Dey, M. Meghjani, F. Shkurti, I. Rekleitis, and G. Dudek, in IEEE/RSJ International Conference on Intelligent Robots and Systems, 2011 (IEEE, Piscataway, 2011), p. 5048.

[16] N. Leonard, D. Paley, F. Lekien, R. Sepulchre, D. Fratantoni, and R. Davis, Proc. IEEE 95, 48 (2007).

[17] S. Dubowsky, K. Iagnemma, S. Liberatore, D. M. Lambeth, J. Plante, and P. J. Boston, in Proceedings of the Space Technology and Applications International Forum-STAIF 2005, edited by M. S. El-Genk and M. J. Bragg, AIP Conf. Proc. No. 746 (American Institute of Physics, Melville, 2005), p. 1449.
[18] M. Duarte, V. Costa, J. Gomes, T. Rodrigues, F. Silva, S. M. Oliveira, and A. L. Christensen, PLoS ONE 11, 1 (2016).

[19] P. Nirmal and D. Lyons, Robotica 34, 2741 (2016).

[20] R. Möller, D. Lambrinos, T. Roggendorf, and R. P. R. Wehner, in Biorobotics: Methods and Applications, edited by B. Webb and T. R. Consi (AAAI, Palo Alto, 2001), pp. 37-66.

[21] A. Mikhailov and D. Meinköhn, in Stochastic Dynamics, edited by L. Schimansky-Geier and T. Pöschel (Springer, Berlin, 1997), pp. 334-345.

[22] P. Romanczuk, M. Bär, W. Ebeling, B. Lindner, and L. Schimansky-Geier, Eur. Phys. J. Spec. Top. 202, 1 (2012).

[23] R. Garcia, F. Moss, A. Nihongi, J. R. Strickler, S. Göller, U. Erdmann, L. Schimansky-Geier, and I. M. Sokolov, Math. Biosci. 207, 165 (2007).

[24] P. Romanczuk, I. D. Couzin, and L. Schimansky-Geier, Phys. Rev. Lett. 102, 010602 (2009).

[25] J. Nötel, I. M. Sokolov, and L. Schimansky-Geier, J. Phys. A: Math. Theor. 50, 034003 (2017).

[26] P. D. Ditlevsen, Phys. Rev. E 60, 172 (1999).

[27] D. Schertzer, M. Larchevêque, J. Duan, V. Yanowsky, and S. Lovejoy, J. Math. Phys. 42, 200 (2001).

[28] J. Noetel, V. L. S. Freitas, E. E. N. Macau, and L. SchimanskyGeier, arXiv:1807.11261v1.

[29] F. J. Sevilla and L. A. Gómez Nava, Phys. Rev. E 90, 022130 (2014).

[30] F. J. Sevilla and M. Sandoval, Phys. Rev. E 91, 052150 (2015). 